Review paper

\title{
Micro-CT of rodents: State-of-the-art and future perspectives
}

\author{
D.P. Clark, C.T. Badea \\ Center for In Vivo Microscopy, Department of Radiology, Duke University Medical Center, Box 3302, Durham, NC 27710, USA
}

\section{A R T I C L E I N F O}

\section{Article history:}

Received 31 March 2014

Received in revised form

15 May 2014

Accepted 28 May 2014

Available online 26 June 2014

\section{Keywords:}

Micro-CT

X-ray

Rodents

Heart

Tumors

Lung

Bone

Nanoparticles

\begin{abstract}
A B S T R A C T
Micron-scale computed tomography (micro-CT) is an essential tool for phenotyping and for elucidating diseases and their therapies. This work is focused on preclinical micro-CT imaging, reviewing relevant principles, technologies, and applications. Commonly, micro-CT provides high-resolution anatomic information, either on its own or in conjunction with lower-resolution functional imaging modalities such as positron emission tomography (PET) and single-photon emission computed tomography (SPECT). More recently, however, advanced applications of micro-CT produce functional information by translating clinical applications to model systems (e.g. measuring cardiac functional metrics) and by pioneering new ones (e.g. measuring tumor vascular permeability with nanoparticle contrast agents). The primary limitations of micro-CT imaging are the associated radiation dose and relatively poor soft tissue contrast. We review several image reconstruction strategies based on iterative, statistical, and gradient sparsity regularization, demonstrating that high image quality is achievable with low radiation dose given ever more powerful computational resources. We also review two contrast mechanisms under intense development. The first is spectral contrast for quantitative material discrimination in combination with passive or actively targeted nanoparticle contrast agents. The second is phase contrast which measures refraction in biological tissues for improved contrast and potentially reduced radiation dose relative to standard absorption imaging. These technological advancements promise to develop micro-CT into a commonplace, functional and even molecular imaging modality.
\end{abstract}

(c) 2014 Associazione Italiana di Fisica Medica. Published by Elsevier Ltd. All rights reserved.

\section{Introduction}

The availability of micro-CT imaging has increased over the last decade and has shown its utility in many preclinical applications. The micro-CT instruments have evolved from custom-made to commercially available scanners designed for either ex vivo or in vivo imaging. Relative to other imaging methods, the strengths of micro-CT lie in its high resolution, relatively low cost, and scanning efficiency. In essence, a micro-CT scanner is based on the same physical principles as a clinical CT scanner, but it is designed for higher-resolution imaging.

A schematic of the complete micro-CT imaging process is shown in Fig. 1. Micro-CT typically produces three-dimensional (3D) tomographic data at microscopic resolution (voxel size $\leq 100 \mu \mathrm{m}^{3}$ ) by taking several hundred, two-dimensional (2D) cone-beam projections from multiple angles around the animal [1]. The raw

\footnotetext{
* Corresponding author. Tel.: +1919684 7509 .

E-mail address: Cristian.Badea@duke.edu (C.T. Badea).
}

projection data are stored on a computer, where they are preprocessed prior to image reconstruction using dark current and flat-field images. The set of log-transformed projection images, also referred to as the cone-beam, X-ray transform of the linear attenuation coefficients, are the input to a tomographic reconstruction algorithm such as the Feldkamp algorithm [2]. The geometric parameters of the scanning are also incorporated into the reconstruction algorithm to produce tomographic images free from misalignment artifacts [3]. The intensity of each voxel within the reconstruction is proportional to the mean linear attenuation coefficient in the specimen at the same spatial location. Reconstructing isotropic voxels allows visualization in any orientation as 2D slices or a rendered 3D volume. This paper is a review of stateof-the-art micro-CT for rodent imaging. We present fundamental principles, relevant technologies, and established applications before introducing new developments associated with spectral and phase contrast imaging. These new developments promise to extend micro-CT imaging as functional and molecular imaging modality. 


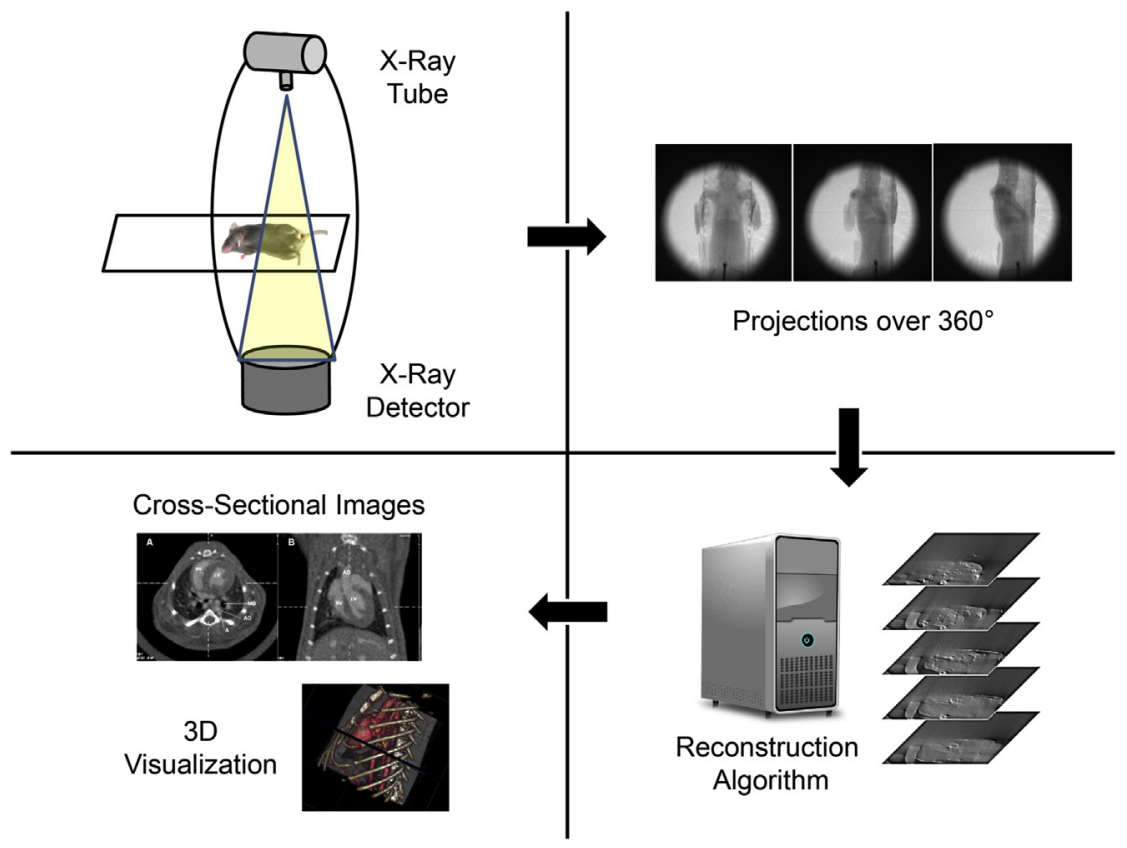

Figure 1. Schematic of the micro-CT imaging process with image acquisition of cone beam projections and reconstruction and visualization of tomographic data.

\section{Technology}

\section{$X$-ray sources}

The choice of the X-ray source strongly affects micro-CT system performance. Due to the tradeoff between focal spot size and thermal loading of the source's metallic anode, most X-ray tubes with mini-focus or micro-focus tubes (focal spot diameter: $<\sim 50 \mu \mathrm{m}$ ) operate with very low photon output (on the order of 100-times lower) compared to the high-power tubes used in clinical scanners [4]. This fact leads to a dramatic increase in the average scan time required in micro-CT to get within an order of magnitude of the noise level seen in clinical CT of about 5-10 Hounsfield units (HU).

Among the different types of X-ray sources for micro-CT found in the literature, the most used are micro-focus, fixed tungsten anode tubes operating in continuous mode, with voltages in the range of $20-100 \mathrm{kV}$ and anode currents in the range of $50-1000 \mu \mathrm{A}$. In vivo micro-CT systems working at low magnification often use pulsed X-ray sources with higher power and wider focal spots, in the range of $0.3-0.6 \mathrm{~mm}$. The use of pulsed X-ray sources is especially important for prospective electrocardiogram (ECG) gating [5]. This type of imaging can also be done with sources operating in continuous mode by using external shutters; nevertheless, the total number of photons emitted during each pulse is very low when using shutters, and the X-ray transmission through the closed shutter must be taken into account for proper raw data calibration.

Additional X-ray tube designs have been proposed. Cao et al. have investigated the use of a compact field-emission micro-focus $\mathrm{X}$-ray source based on carbon nanotubes [6,7]. In this type of source, the metal filament cathode is replaced by a field emission cathode that is capable of emitting electrons at room temperature with voltage controlled output current [8]. The authors obtained stable emission of X-rays at $50 \mathrm{kV}$ and $1 \mathrm{~mA}$, with a pulse length down to $20 \mathrm{~ms}$ and with a focal spot size of 100 microns. Despite the improvement of a factor of 5-6 in focal spot size with respect to standard pulsed X-ray sources, the overall performance of the resulting micro-CT scanner in terms of spatial resolution was comparable to that of other systems for in vivo imaging $(5 \mathrm{lp} / \mathrm{mm}$ at $10 \%$ of the modulation transfer function, MTF) because of the low magnification used. We note that the MTF measures contrast transfer from the object to the reconstructed image as a function of spatial resolution. The $10 \%$ cutoff (i.e. the spatial resolution at which $10 \%$ of the contrast is transferred) is often used as a measure of spatial resolution in CT systems (reported in line pairs/distance).

For ex vivo imaging of small biological samples at very high magnification, the need for very small focus is more stringent because it represents the main limiting factor of the spatial resolution. Reflection-anode X-ray tubes with focal spots in the range of 5-20 microns are available on the market. Because of the very high power density that such narrow electron beams can release on the target surface, the anode current must be controlled as a function of focal spot size to prevent melting [9]. The maximum anode currents in most micro-focus X-ray tubes are on the order of $100-200 \mu \mathrm{A}$, with maximum power $<10 \mathrm{~W}$.

Other drawbacks of reflection-type X-ray sources with thick targets include a relatively narrow aperture of the cone beam (in the range of $30^{\circ}-60^{\circ}$ ) and a limited capability of reducing the focus-toobject distance below a few millimeters. Non-uniformity of the beam intensity due to the heel effect can be reduced by flat-field (gain) correction; however, accounting for the variation in the Xray spectrum across the beam profile is difficult [10]. Open-type sources with thin-target transmission anodes overcome these limitations, providing radiation beams with angular apertures in the range of $140^{\circ}-160^{\circ}$ with the possibility of putting the object virtually in contact with the focus. However, the back of transmission targets cannot be cooled with liquid, reducing the maximum possible power per unit area compared to reflection anodes. The spatial resolution obtainable with this type of source can be $<1$ micron, which is similar to synchrotron based imaging. Nevertheless, synchrotron-based micro-CT and nano-CT are still superior to laboratory-level micro- and nano-CT systems in terms of contrast resolution and signal-to-noise ratio because synchrotrons provide tunable, monochromatic radiation, avoiding beam hardening artifacts, and much higher photon fluxes, reducing noise [11-13]. 


\section{$X$-ray detectors}

The X-ray detector is a key component of a micro-CT scanner. The majority of micro-CT systems to date employ digital flatsurface 2D detectors leading to a cone beam scanning geometry. Apart from a single case known in the literature of high-resolution CT reconstruction of a rat kidney from the digitization of multiple film radiographs [14], early prototype micro-CT systems employed $\mathrm{X}$-ray image intensifiers read by charge-coupled devices (CCDs) [15-17]. Later in the mid-1990s, combined detection systems made up of scintillator screens coupled to CCDs via fiber-optic bundles, with various de-magnifying ratios, became the standard for microCT imaging $[18,19]$. More recently, advances in complementary metal oxide semiconductor (CMOS) technology led to the production of large area detectors with high frame rates which are the most widely used systems for in vivo, small animal imaging [20,21]. Cooled CCDs are still in use, especially in applications involving low $\mathrm{X}$-ray fluences, due to their very low dark noise with respect to flatpanel CMOS detectors. Also reported in the literature are studies on the use of direct conversion detectors coupled to thin-film transistor (TFT) arrays [19] and of single-pixel or small area detectors operating in photon counting mode $[18,22,23]$ for small animal micro-CT.

Energy discriminating photon counting X-ray detectors (PCXD) are the subject of intensive research and promise to make spectral CT a reality. In fact, micro-CT is the testing stage for spectral CT using PCXD [24]. PCXDs with energy binning can improve CT performance by counting and binning each X-ray detected into a number of energy bins equal to the number of energy thresholds per pixel. PCXDs allow for the elimination of dark noise in the image by rejecting all counts below the signal and also allow for spectral separation. The technology, currently in its infancy, is set to grow rapidly.

Sensor materials for PCXDs include silicon (Si), gallium arsenide (GaAs), and cadmium telluride (CdTe) with pixel sizes as small as $55 \mu \mathrm{m}$ [24]. Yet, there remain technical limitations to spectral detectors that preclude their immediate replacement of conventional, energy-integrating detectors for biomedical CT applications [25]. For example, $\mathrm{Si}$ is an X-ray detector material with fast signal collection and low susceptibility to signal pile-up but suffers from a high fraction of Compton interaction at X-ray energies encountered in CT practice, degrading its spectral imaging capabilities [26]. For example, the Medipix detector has a small pixel size and high spatial resolution and is better suited for imaging of small animals in preclinical applications than other detectors [24,27]. Medipix is a series of photon-counting detectors for X-ray micro-imaging from the CERN [28]. Medipix1 has a pixel size of $170 \times 170 \mu \mathrm{m}$ Medipix2 reduces the pixel size to $55 \times 55 \mu \mathrm{m}$. The performance of Medipix2 is limited by charge sharing over neighboring pixels, compromising energy resolution. Medipix3 addresses this problem using a photon-processing chip with special circuitry to allow charge deposition in adjacent pixels to be summed and analyzed with two simultaneous energy thresholds and without spectral distortion [27]. The readout logic supports eight energy thresholds over $110 \times 110 \mu \mathrm{m}$ for spectroscopic imaging. A commercially available micro-CT system (MARS; University of Canterbury; Christchurch, New Zealand) is equipped with Medipix3 detectors. We review spectral micro-CT in Section Spectral micro-CT.

\section{System geometry}

There are two common system geometries for micro-CT: rotating gantry and rotating specimen. Most in vivo micro-CT systems employ a rotating gantry geometry in which the X-ray tube and detector are assembled in a single gantry. The gantry rotates around a central axis while the animal or the specimen lies stationary on a table between the tube and detector (Fig. 1). By contrast, most ex vivo micro-CT systems employ a rotating specimen geometry where the X-ray source and detector are stationary. For rotating specimen systems, changing the position of the specimen to adjust magnification can be more practical than in rotating gantry systems. The rotating specimen geometry has not gained popularity for in vivo imaging, primarily because the animal must be mounted vertically.

In designing micro-CT systems with either geometry, a balance must be struck between the system dimensions, the required spatial resolution, the focal spot size of the X-ray source, and the photon fluence produced by the X-ray source (Section X-ray sources). Positioning the specimen closer to the X-ray source and farther from the detector increases geometric magnification, increasing spatial resolution while reducing the field of view covered by the detector. The magnification can be increased up to the limits imposed by the system dimensions or by penumbra blurring. Penumbra blurring describes the X-ray projection blur resulting from the finite size of the X-ray tube focal spot and the system configuration [29]. If the specimen to detector distance and source to specimen distance are similar, as in most rotating gantry systems, penumbra blurring becomes comparable to the focal spot size. Rotating gantry systems operating at moderate magnifications for dynamic, in vivo micro-CT imaging include [30] and [31]. To allow the use of a high fluence X-ray source with a large focal spot, the specimen can be placed close to the detector, limiting spatial resolution, but enabling very short exposures for in vivo cardiorespiratory imaging in rodents. This configuration has been demonstrated for dynamic, in vivo micro-CT imaging in a rotating specimen geometry [32].

Regardless of system geometry, a very important requirement for successful image reconstruction is geometric calibration [3]. Some scanners now provide zoom-in capability and variable geometry [33]. With such changes, geometric calibration is required to maximize spatial resolution in the reconstructed image data. Automated calibration methods have been developed which estimate geometric parameters based on projections of a generic object [34] and by reconstruction-based optimization of image sharpness metrics [35].

\section{Image reconstruction}

CT image reconstruction solves an inverse problem which maps cone beam projection data to a 3D matrix corresponding to a discretized, tomographic representation of the specimen [36]. There are two major types of algorithms: 1) filtered back projection (FBP)based algorithms, and 2) iterative algorithms [37]. The most commonly used reconstruction algorithms in CT and micro-CT are based on FBP. In FBP, the acquired, 2D, cone-beam projections are filtered, using a convolution kernel that reduces the blurring inherent to the back-projection process, and then they are backprojected (i.e. smeared back) through the object space at the appropriate angle to generate an image. While first developed for 2D imaging (parallel or fan beam), convolution back projection was adapted by Feldkamp et al. [2] to compensate for the cone-beam geometry. This method forms the basis for the reconstruction algorithms used in most current micro-CT systems. While Feldkamp's algorithm is considered to be an approximation, since the circular sampling trajectory does not satisfy Tuy's data sufficiency condition [38], the quality of the reconstructed images is acceptable if the cone angle is less than about $10^{\circ}$ [1], except for very unusual object configurations [39].

When the number of projections available for reconstruction is limited and/or the projections are very noisy (e.g. low dose 
a) FBP
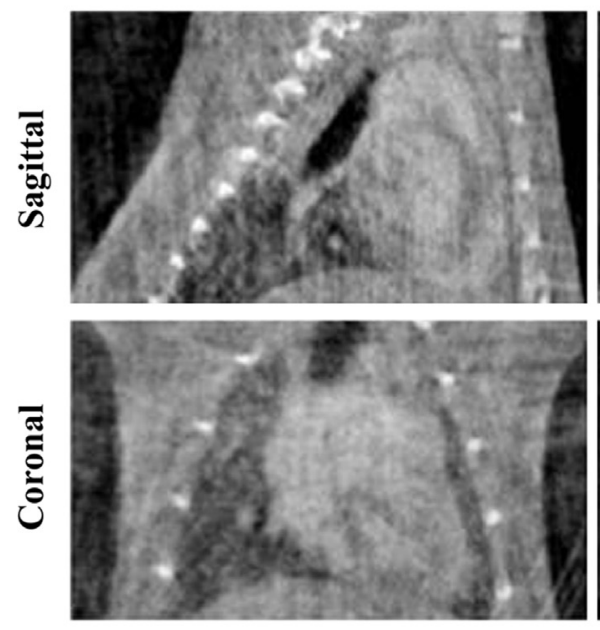

b) SART-TV
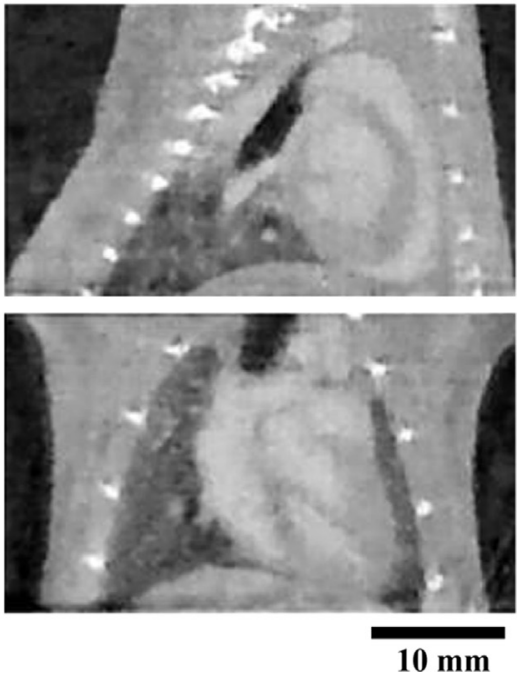

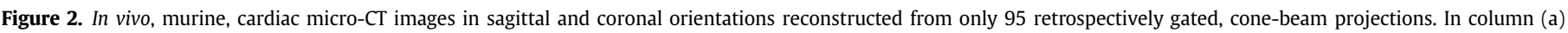

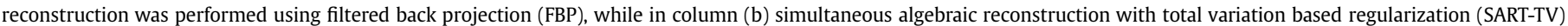
was used. Note the decrease of artifacts in column (b).

scanning), iterative and statistical reconstruction algorithms can provide notably better image quality than FBP. Reduction in the number of views translates directly to reduced radiation dose to the animal. Furthermore, it is often impractical to acquire projections with regular-angular sampling in dynamic imaging applications (Section Cardio-respiratory gating in small animal micro-CT), resulting in reconstruction artifacts when FBP is used. Iterative algorithms such as the algebraic reconstruction technique (ART) [40] present an alternative to FBP with the potential for robust reconstruction given less than ideal projection data [37]. With such algorithms, the reconstructed volume is refined by repeatedly comparing simulated re-projections of the reconstructed volume with the original projections.

Artifacts from FBP reconstruction with an irregular-angular distribution of projections manifest as long thin streaks and shading artifacts. Total variation minimization (TV) has been recognized for its ability to suppress artifacts, while leaving boundaries between homogeneous regions intact [41]. TV minimization can be interleaved with iterative reconstruction algorithms such as the simultaneous algebraic reconstruction technique (SART) by performing one or more iterations of each in turn. To illustrate the power of SART-TV based reconstruction using irregularly sampled projections, we present its application in 4D cardiac micro-CT. Specifically, Fig. 2 compares image quality of FBP (a) and SART-TV (b) reconstructions when only 95 irregularly sampled projections were used [42]. Note the reduction of the streaks with SART-TV.

Statistical image reconstruction is another class of iterative reconstruction algorithms, first introduced for transmission imaging in nuclear medicine, but also applied for CT reconstruction [43]. In essence, these algorithms treat the reconstruction as a statistical estimation problem and have the advantage that they can accurately model Poisson noise in the projection data. This results in lower noise levels with statistical reconstruction for the same data when compared with FBP reconstruction.

One major limitation of iterative reconstruction algorithms is their running time. To overcome this limitation, the graphics processing unit (GPU) has been recognized as an alternative computer architecture with potential for accelerating CT reconstruction [44]. This parallel architecture is ideal for CT reconstruction steps in which the same arithmetic operations must be performed at multiple pixels or voxels (e.g. projection, back projection). The development of programming tools for general-purpose computing on the GPU, such as NVIDIA's Compute Unified Device Architecture (CUDA; Santa Clara, CA), has facilitated the acceleration of CT reconstruction, presenting the opportunity to explore more complex iterative algorithms. Micro-CT is already benefiting from GPUbased implementations of analytical, iterative, and statistical image reconstruction [45-47].

\section{Cardio-respiratory gating in small animal micro-CT}

The influence of cardiac and respiratory motion during in vivo scanning can be minimized by using cardio-respiratory gating. 4D micro-CT used in cardio-pulmonary studies typically employs either prospective gating (PG) or retrospective gating (RG). In PG, acquisition is triggered by the coincidence of a selected respiratory phase and a selected cardiac phase. This produces a set of projections with a constant angular step, resulting in reconstructed images that are free of streaking artifacts. However, because of the time spent waiting for the coincidence of cardiac and respiratory events, the scan time can take as long as $1 \mathrm{~h}$ to cover 10 different phases of the cardiac cycle [4].

In $\mathrm{RG}$, the projection images are acquired at a rapid and constant rate without waiting for cardiac and respiratory coincidence. Respiratory and ECG signals are monitored and saved in synchrony with the acquisition of the projections. Using these signals postsampling (i.e. retrospectively), the projections are sorted into different subsets corresponding to different cardiac and respiratory phases. With this protocol, the scan time can be shortened to $50 \mathrm{~s}$, when using a slip ring gantry [30]. However, the irregular angular distribution causes streaking artifacts in the FBP-based reconstructed images. As shown by Fig. 2, superior results are possible using regularized iterative algorithms such as SART-TV [42]. More recently, a new gating strategy called fast prospective gating (FPG) has been introduced [48], which combines the regular angular distribution of PG with the fast scan time of RG. In FPG, multiple projections are acquired at the same angle, corresponding to all cardiac or respiratory phases to be reconstructed, before the cradle is rotated to the next angle. FPG requires on-the-fly computation of 
the triggering events, which are delayed from the peaks of the respiratory or cardiac signals.

In terms of implementation, most of the proposed methods involve extrinsic cardio-respiratory gating, in which the cardiac and respiratory signals are acquired with dedicated monitoring devices (i.e. ECG leads and a pneumatic respiratory pillow). In addition to these extrinsic gating techniques, an intrinsic image-based gating approach without any external devices was developed, initially for a clinical, spiral cone-beam CT scanner [49]. In intrinsic gating, a post-processing algorithm evaluates the center of mass of certain regions of interest (ROIs) within each projection to detect respiratory and cardiac motion [50-52].

\section{Contrast agents}

One of the major challenges for CT imaging is its poor contrast sensitivity in biological soft tissue. Several staining methods have been proposed to increase soft tissue contrast in ex vivo specimens scanned with micro-CT. Osmium staining has been demonstrated successfully in several micro-CT applications, including phenotyping mouse embryos [53]. Other stains, based on inorganic iodine and phosphotungstic acid, are easier to handle and much less toxic than osmium, and they produce high-contrast X-ray images of a wide variety of soft tissues $[54,55]$.

Vascular contrast agents for in vivo micro-CT imaging fall into one of two categories: (1) low molecular weight and (2) blood pool. Iodine-based, low molecular weight contrast agents used for clinical CT imaging applications (e.g. Omnipaque from GE Healthcare, Isovue from Bracco Diagnostic) can also be used for preclinical micro-CT in rodents; however, such agents clear from mouse vasculature within seconds [56]. Iodine-based, blood pool contrast agents (e.g. Fenestra from ART, eXIA from Binitio Biomedical) provide stable enhancement over the course of minutes to an hour [57], facilitating translation of clinical imaging applications to rodent model systems. Larger nanoparticle contrast agents based on variety of high atomic number elements show great potential for functional and molecular CT imaging applications and provide fine control over vascular half-life. Examples include $100 \mathrm{~nm}$ liposomes encapsulating iodine [58] (half-life: $40 \mathrm{~h}$ in mice) or gadolinium [59], 15 nm pegylated gold nanoparticles (AuroVist from Nanoprobes, Inc., half-life: $\sim 15 \mathrm{~h}$ in mice), and barium-based ExiTron nano from Miltenyi Biotec.

Nanoparticle-based contrast agents have now opened the door for molecular CT imaging using various passive and active targeting strategies [60-65]. Such probe development is extremely important for the future of spectral micro-CT, extending its capabilities to provide functional and molecular information [66]. For example, fibrin-targeted nanoparticles have been used to detect and characterize atherosclerotic plaques [67]. Current and future applications of blood pool and nanoparticle contrast agents for in vivo micro-CT imaging are discussed at length in Section Applications and Section Spectral micro-CT.

\section{Radiation dose}

X-ray exposure can be harmful since it can disrupt chemical bonds and can create free radicals in the body [68,69]. Micron-scale spatial resolution requires relatively high radiation exposure, often making radiation dose a concern and limitation for in vivo applications [68]. The total absorbed radiation dose is a function of radiation exposure from the X-ray tube (measured in milliampereseconds), beam hardness, and desired image quality (resolution, noise, and contrast) [70]. Increasing the spatial resolution for in vivo micro-CT imaging is non-trivial due to the associated increase in radiation dose. To illustrate, using an ideal micro-CT scanner, a coefficient of variation (defined as the ratio between the mean value and noise) with a value of $1 \%$ can be obtained at $135 \mu \mathrm{m}$ resolution and a dose of $0.25 \mathrm{~Gy}$. However, if the resolution is to be increased to $65 \mu \mathrm{m}$, the associated dose for the same coefficient of variation would be nearly 5 Gy $[68,71]$.

One way to express dose limitations for small animals is in terms of the lethal dose (LD) for a population. $\mathrm{LD}_{50 / 30}$ refers to the wholebody radiation dose that would kill $50 \%$ of the exposed animals within 30 days. The $\mathrm{LD}_{50 / 30}$ in mice is reported to range between 5 and 7.6 Gy depending on the strain of mouse, age at exposure, and many other factors [72-75]. Typical X-ray, whole-body radiation dose for a 3D micro-CT scan reported in the literature ranges from $0.017 \mathrm{~Gy}-0.78 \mathrm{~Gy}$, depending on the diagnostic demand and the contrast resolution required [76].

If micro-CT is performed longitudinally, radiation dose can accumulate. The thorax and abdomen are the most radiosensitive regions of an animal, with the most immediate symptom of highdose micro-CT being radiation-induced pneumonitis. Pneumonitis can arise as a lethal complication when the thorax is exposed to over $1.9 \mathrm{~Gy}$ [77]. Fortunately, rodents have the ability to repair sublethal doses of radiation over periods of a few hours, so that repeated exposures of about 30 cGy have not compromised the survival of mice in previous studies [78]. Taschereau et al. have reported that the average whole-body dose for longitudinal microCT exams is likely to be less than $16 \mathrm{cGy}$ [70], making it unlikely that radiation exposure will be a limiting factor for longitudinal studies using in vivo micro-CT. In fact, in a recent study, C57BL/6 mice were imaged with micro-CT multiple times for 6 weeks in order to determine the effect of the cumulative dose on pulmonary and cardiac tissue at the end of the study [79]. The study concluded that a dose as high as $5 \mathrm{~Gy}$ accumulated over 6 weeks during a longitudinal micro-CT study had no significant effects on the pulmonary and myocardial tissue of $\mathrm{C} 57 \mathrm{BL} / 6$ mice. However, the radiation dose associated with in vivo micro-CT imaging protocols should be estimated and minimized whenever possible. Such considerations are especially crucial in cancer therapy applications where the radiation dose associated with imaging must be sub-therapeutic to avoid complicating interpretation of the results.

\section{Applications}

\section{Bone imaging}

Micro-CT has been used to investigate the structure and density of rodent bone since its very beginnings $[15,80]$, due to its high spatial resolution and high contrast in imaging mineralized tissues. In fact, the study of bone architecture and density drove the early developments of micro-CT systems [81]. When employed in ex vivo studies, the spatial resolution of dedicated bench-top systems approaches that provided by synchrotron sources [82]. Since the acquisition protocol is constrained by dose to the animal [68], in vivo acquisitions produce reconstructions with lower spatial resolution. Nonetheless, a nominal spatial resolution of about $50 \mu \mathrm{m}$ has been shown effective in following live rodents during studies of osteoarthritis [83,84] and bone remodeling [85].

The application of micro-CT in longitudinal, orthopedic studies has been demonstrated in the study of bone architecture changes over time [86,87], where parameters such as volumetric bone mineral density, bone volume ratio, bone surface ratio, and trabecular thickness are reported. A comprehensive review of the morphometric parameters that can be extracted from bone microCT images has been published [88]. Such micro-CT bone structural parameters were used to quantify the effects of vascular endothelial growth factor (VEGF) [89] and stem cell treatments [90] in bone healing and also bone loss and cortical thickening in total body 


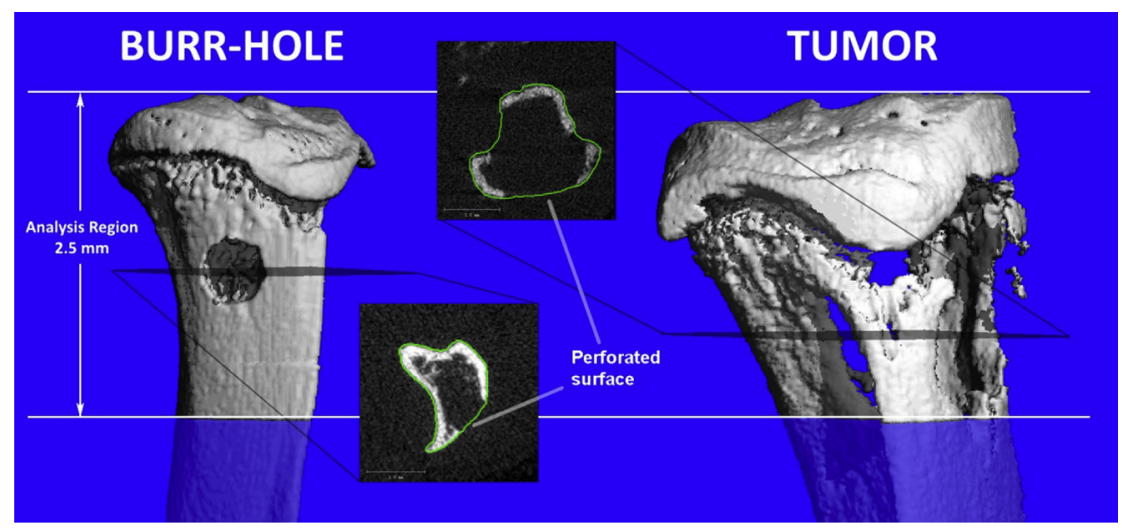

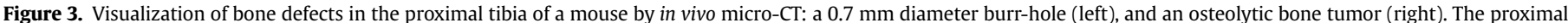

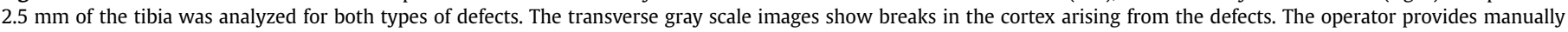
drawn contours approximating an intact bone for the analysis. Figure reproduced from Ref. [105] with permission.

irradiation and bone marrow transplantation in mice [91]. In osteoporosis research, micro-CT data of the proximal tibiae in small animal models enables study of the progression of disease after ovariectomy [92] or immobilization [93]. Similar quantification has been performed on human biopsy specimens from iliac crest bone to assess the effect of therapy via injection of human parathyroid hormone [94]. In bone regeneration studies, the calvarial defect model in rats, which can provide quantitative information on bone mineral content and bone fractional area [95], has been used extensively with micro-CT imaging. Also in the field of regenerative medicine, micro-CT plays a fundamental role in the accurate determination of the three-dimensional structure of engineered bone scaffolds, with particular emphasis on porosity and connectivity [96,97]. Finally, the use of finite element methods (FEM) applied on computerized meshes generated from segmented bone images allows the nondestructive evaluation of the mechanical properties of bones and scaffolds, improving understanding of bone biomechanical properties and reducing the number of sacrificed animals [98].

Some applications, such as studies of skeletal development [99], may accept lower resolution but require a larger field of view, i.e. imaging the whole mouse or rat using a flat-panel detector $[30,100,101]$. The study of cancelous bone requires higher resolution, and is often performed ex vivo using excised bones. This is due to the thickness of trabecule in small animal cancelous bone, which can be as thin as 30-50 microns [102]. To avoid quantification errors due to the partial volume effect, voxel sizes in the range of 1-20 microns are used for this type of application. As a rule of thumb, the imaging system's full width at half maximum (FWHM) spatial resolution should be no more than half the minimum thickness to be measured to mitigate the influence of partial volume effects.

The quantification of the morphometric parameters is performed on a binary image obtained by segmenting the original micro-CT volume. In most cases, due to the high contrast between bone, marrow, and muscle, simple threshold-based segmentation is sufficient for both cancelous and cortical bone segmentation on micro-CT images of adequate spatial resolution. In some cases, the original images must be corrected for non-uniformity in order to reliably apply a single global threshold. In all cases, care must be taken in the selection of the threshold value, as it has a significant impact on the results [103]. Several automatic methods have been developed for the robust selection of the threshold value, mostly based on analysis of a histogram of image intensities [104].

Figure 3 illustrates a bone study using in vivo micro-CT [105]. The study demonstrates that measures of inter-microarchitectural bone spacing are sensitive to the presence of focal defects in the proximal tibia for two distinctly different mouse models: a burrhole model for fracture healing research, and a model of osteolytic bone metastases. In these models, the cortical and trabecular bone compartments were both affected by the defect and were, therefore, evaluated as a single unit.

\section{Lung imaging}

Enabled by the inherent contrast between air and tissue, CT is a powerful modality for lung imaging. However, pulmonary microCT imaging in small animals has been challenging due to their small size and rapid respiratory motion. A voxel size on the order of $75 \mu \mathrm{m}$ is required to provide anatomic resolution in the mouse comparable to that obtained in CT for humans [106]. Micro-CT can be used successfully to study various lung-disease models, such as emphysema and fibrosis. An emphysema model was created in mice by using intra-tracheal instillation of pancreatic elastase and imaged with micro-CT [107]. Emphysemic regions have shown lower HU values than the normal lung, while the lung volume in these diseased animals was increased. Fibrosis models also have been created in mice by instillation of bleomycin and studied longitudinally with micro-CT during diseases progression [108]. The fibrotic lung regions are identified in micro-CT images by the resulting increase in HU. Furthermore, some studies have also shown that lung compliance can be measured in small animals using micro-CT, by implementing breath-holding over a range of specified pressures and calculating lung volumes from the corresponding images to produce a pressure-volume curve $[108,109]$. Micro-CT imaging-based compliance measurements were validated against the gold standard provided by quasi-static compliance measurements using a commercially available small animal ventilator [108]. The loss of pulmonary compliance was investigated in a study of irradiated mice [110].

Figure 4 presents color-coded images from a study focused on the detection and early phase assessment of radiation-induced lung injury in mice [111]. A very early response (1-4 days postirradiation) of the lung to radiation exposure was detectable as $\mathrm{HU}$ alterations on 3D CT images. Areas of high density (between -200 to $-500 \mathrm{HU}$ ) in 3D micro-CT slices are shown in green, and areas of low density (below $-500 \mathrm{HU}$ ) are shown in blue. The study concluded that both micro-CT and histomorphometry give reliable measurements of air space enlargement associated with early phase, radiation-induced lung injury in mice. However, micro-CT offers advantages, by enabling longitudinal studies and 

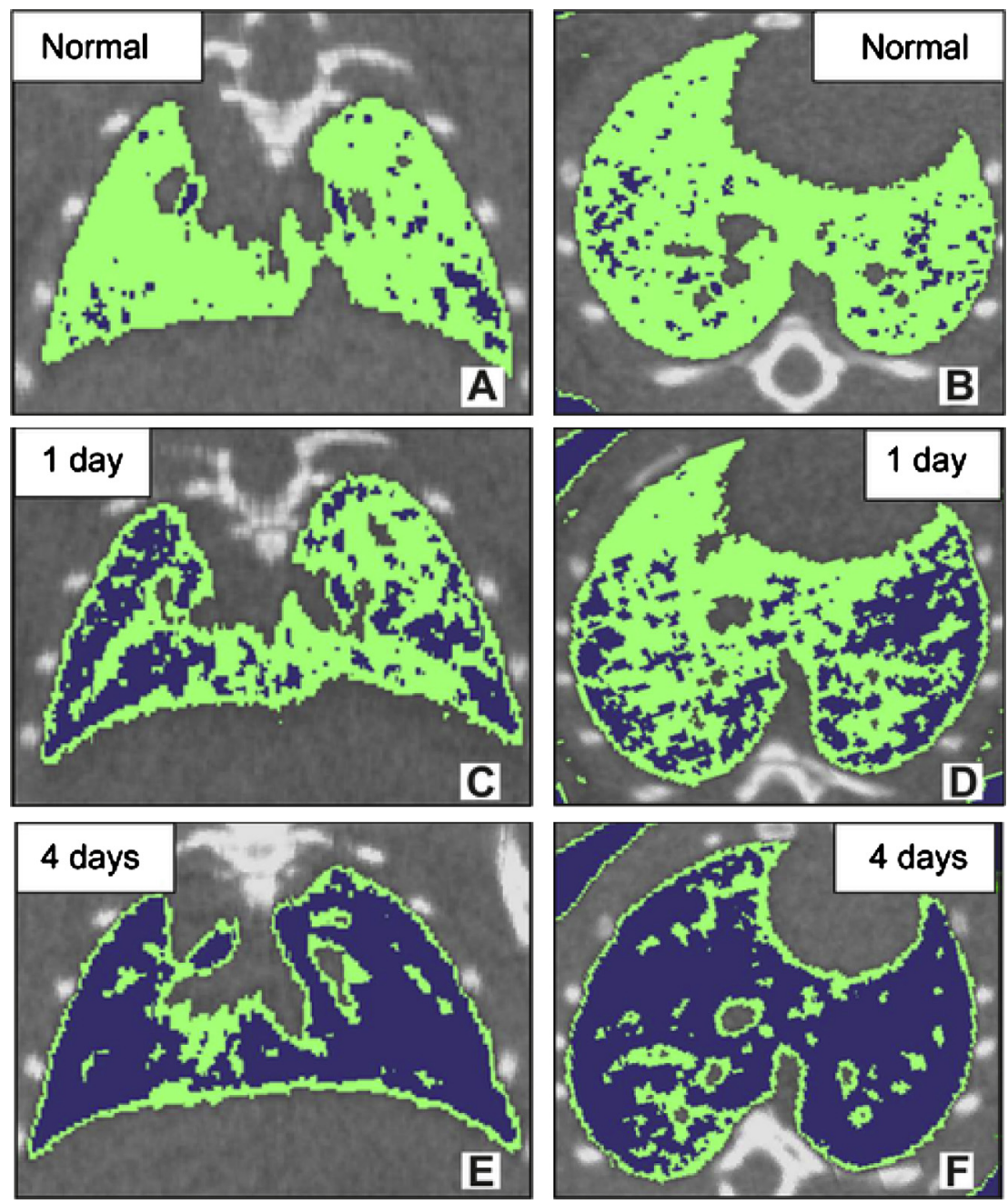

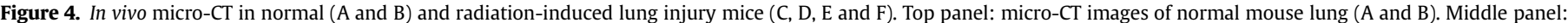

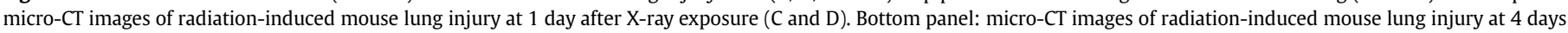

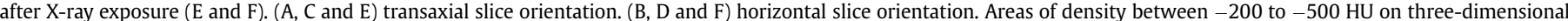

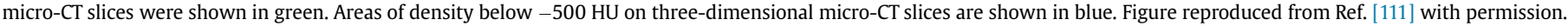
(For interpretation of the references to color in this figure legend, the reader is referred to the web version of this article.)

providing a three-dimensional analysis of the entire lung, thus reducing the number of animals required for studies and providing more relevant information on the progression of lung disease.

In vivo micro-CT also provides the capability for contrastenhanced imaging of the rodent lung using stable xenon gas as an inhaled contrast agent. This procedure has been described in a study with adult rats [112].

\section{Cardiac imaging}

Micro-CT has been used to visualize the 3D coronary circulation ex vivo in the young and aged rodent heart using radiopaque MICROFIL (FlowTech, Inc., Carver, MA, USA), a silicon-based polymer [113]. 3D phenotyping of cardiovascular development in mouse embryos also has been achieved by ex vivo micro-CT using commercially available iodine-based contrast agents with low toxicity, and low cost [114]. In vivo micro-CT based cardiac morphological and functional imaging in mice and rats has been reported using both PG and RG approaches [30-32,42,115-118].

Cardiac micro-CT requires the use of contrast agents to be able to discriminate between the myocardium and blood. In most studies, blood-pool contrast agents have been used, although, using low molecular weight contrast agents is also possible with slip ring technology and flat panel detectors [30,31]. For example, recently, a method for imaging cardiac perfusion in the mouse has been proposed using a scan protocol consisting of repetitive injections of conventional, low molecular weight contrast media within several consecutive scans [119]. However, most cardiac micro-CT studies use a blood pool contrast agent and acquire 4D data to measure cardiac functional metrics such as ejection fraction and cardiac output. The accuracy of these measurements was studied as a function of the volume of contrast agent and the number of projections used for reconstruction [117]. Reduction of the volume of contrast agent reduces hypervolemia effects on the hemodynamics, while the reduction in the number of projections translates directly into reduced radiation dose. It was determined that if 5\% error in LV volumetric estimation is acceptable, as suggested for human cardiac CT [120], then sufficient cardiac micro-CT image quality could be provided with approximately 126 projections and a contrast dose of $0.01 \mathrm{ml}$ Fenestra VC (ART Advanced Research Technologies, Inc., Quebec, Canada) per gram body weight. With other formulations of blood pool contrast agents that contain higher iodine concentration, the total volume injected may be further reduced. Formulations such as eXIA 160 (Binitio Biomedical, Inc., Ottawa, 
Canada), an aqueous colloidal poly-disperse contrast agent with a high iodine concentration ( $160 \mathrm{mg} \mathrm{I} / \mathrm{ml}$ ), also have been used for cardiac micro-CT, since it creates strong contrast between blood and tissue with a low injection volume of only 0.005 per gram in a mouse [121].

Global cardiac functional metrics such as cardiac output, stroke volume, ejection fraction, and myocardial mass and localized, dynamic metrics such as wall mall motion can be computed using 4D cardiac micro-CT data sets. Nominally, these measures require laborious segmentation of the murine heart in 4D; however, numerous software packages (Avizo, VSG, Burlington, MA; Vitrea, Vital Images, Minnetonka, MN; ITK-SNAP, [122]) and algorithmic methods [123-125] are available for semi- and fully-automated cardiac segmentation. Efficient, fully-automated cardiac segmentation is highly desirable for functional and structural phenotyping applications. One such phenotyping application imaged transgenic muscle LIM protein null mice to compare the micro-CT measurements with M-mode echocardiography in a model of heart failure [115]. Cardiac micro-CT has also been used for the evaluation of drug effects, such as shown in a study on dobutamine-induced cardiac stress in rats [126].

Cardiac micro-CT can also be used for weekly imaging following a sham or coronary-ligation surgery to monitor left ventricular (LV) remodeling post-infarction [118]. Assessment of cardiac function and infarct size were also demonstrated using delayed contrast enhancement by combining the blood pool contrast agent Fenestra VC and a low molecular weight contrast agent [116]. Infarcts also have been imaged using a novel preclinical contrast agent called EXIA 160 [57] which creates strong contrast between blood and tissue immediately after its injection and is subsequently taken up by the myocardium and other metabolically active tissues over time.

As with all in vivo micro-CT, the dose delivered to the animal must be considered. When PG-based cardiac micro-CT was initially proposed, it involved long scan times and a radiation dose on the order of $1 \mathrm{~Gy}$ [127]. With retrospective gating, the entrance exposure was less than $0.3 \mathrm{~Gy}$ to reconstruct scans covering the entire cardiac cycle [30]. More recent 4D micro-CT methods using superior image reconstruction algorithms have achieved a dose close to 0.2 Gy $[52,128-130]$.

\section{Cancer imaging}

Micro-CT has proven to be useful in tumor detection and tracking, as well as in imaging tumor angiogenesis. For example, micro-CT has been used to detect murine lung nodules with a minimum volume of $0.63 \mathrm{~mm}^{3}$ [131], when using a ventilator and prospective respiratory gating.

Cancer imaging with micro-CT is intimately related to the use of contrast agents. Dynamic micro-CT has been applied to the direct measure of perfusion in tumors following the injection of conventional contrast agent [132,133]. Acquisition of perfusion information requires rapid scanning and has only become possible with the introduction of slip-ring based micro-CT scanners capable of acquiring 3D image data sets once per second for periods of a minute or longer [134]. To achieve adequate coverage in the longitudinal direction, the rapid scans often require a reduction in spatial resolution in the longitudinal direction. CT perfusion measurements enable the quantitative evaluation of functional parameters, such as blood flow, blood volume, and permeabilitysurface area product-a measure of the leakage rate of contrast from the capillaries into the interstitial space. As previously discussed (Section Contrast agents), due to their rapid clearance in small animals, conventional low molecular weight contrast agents are not widely used for micro-CT. Instead, blood pool and nanoparticle contrast agents are preferred for cancer imaging with micro-CT. Next, we present a number of examples of cancer imaging using blood pool and nanoparticle contrast agents.

Using Fenestra VC, Kindlmann et al. [135] examined the intermediate and large vessels of alveolar rhabdomyosarcomas in a transgenic mouse model. The investigators suggested that microCT with Fenestra VC had the best potential for defining vessel diameter, tortuosity, and density for anti-angiogenesis models, but to visualize tumor capillaries, it was necessary to develop instruments that had a higher resolution than those used in their study.

Micro-CT imaging studies were performed in nude mice bearing STC- 1 tumors 15 and 30 days after grafting [136]. Animals were injected with Fenestra LC and imaged at 2 and $4 \mathrm{~h}$ after the injection. The liver and spleen were clearly differentiated after administration of the contrast agent, reaching mean contrast-to-noise ratios of $>2.0$ for the liver and $\sim 10$ for the spleen at the different imaging times. In the spleen, quantification of the tumors with Fenestra LC was not precise, whereas in the liver, tumors (0.3-1.5 mm) were detectable at day 30. Significant amounts of the contrast media were observed to persist in the animals for up to 15 days after a single injection, allowing quantitative follow-up of tumors both in the liver and the spleen, without additional injection of contrast. Strain-specific variations in Fenestra LC uptake and signal duration have been observed in mice without tumors [137].

Liver tumor volumes also have been quantified in vivo using Fenestra VC in mice injected with murine B16F1 melanoma cells; in this case Fenestra VC (Vascular Contrast) was used instead of Fenestra LC (Liver Contrast) in order to remove the ambiguity between unopacified liver vessels and tumors in the liver $8 \mathrm{~h}$ post contrast injection [138]. Because many liver metastases grow on the surface of the liver, a combined strategy of Fenestra VC plus an intraperitoneal injection of extracellular contrast agent was shown to improve tumor volume measurement [138]. Small lesions like liver metastases also have been imaged using a nanoparticle alkaline earth metal-based contrast agent (ExiTron nano from Miltenyi Biotec $\mathrm{GmbH}$ ) developed for small animal liver imaging with $\mathrm{CT}$ [139].

A liposomal, iodinated contrast agent (Lip-I) has been used to image the tumor micro-environment [140,141]. Figure 5 outlines a typical tumor study in which Lip-I is used to measure fractional blood volume based on CT data acquired soon after contrast injection and vascular permeability (extravasation) resulting from the enhanced permeability and retention (EPR) effect [142] at a later time point [140]. The same Lip-I has enabled differentiation between fast- and slow-growing primary lung tumors in mice [143] based on differential accumulation (enhancement) over time. In another study, Lip-I accumulation increased significantly in primary sarcomas after mice received 20 Gy radiation treatment [144].

\section{Multi-modality imaging using micro-CT}

Another way in which micro-CT can be used in the study of both function and anatomy is by combining it with other imaging modalities, such as positron emission tomography (PET), singlephoton emission computed tomography (SPECT), or fluorescence tomography [145]. Although very sensitive, fluorescence tomography and nuclear tomographic imaging methods are limited by poor spatial resolution, which becomes a significant issue when imaging mice. Micro-CT is therefore useful in providing anatomical reference and also enables, in the case of nuclear imaging, attenuation correction. Multi-modality imaging is most efficiently performed by combining both imaging modalities (micro-PET/microCT or micro-SPECT/micro-CT) into a single scanner (sometimes referred to as hardware image fusion) and then by performing 


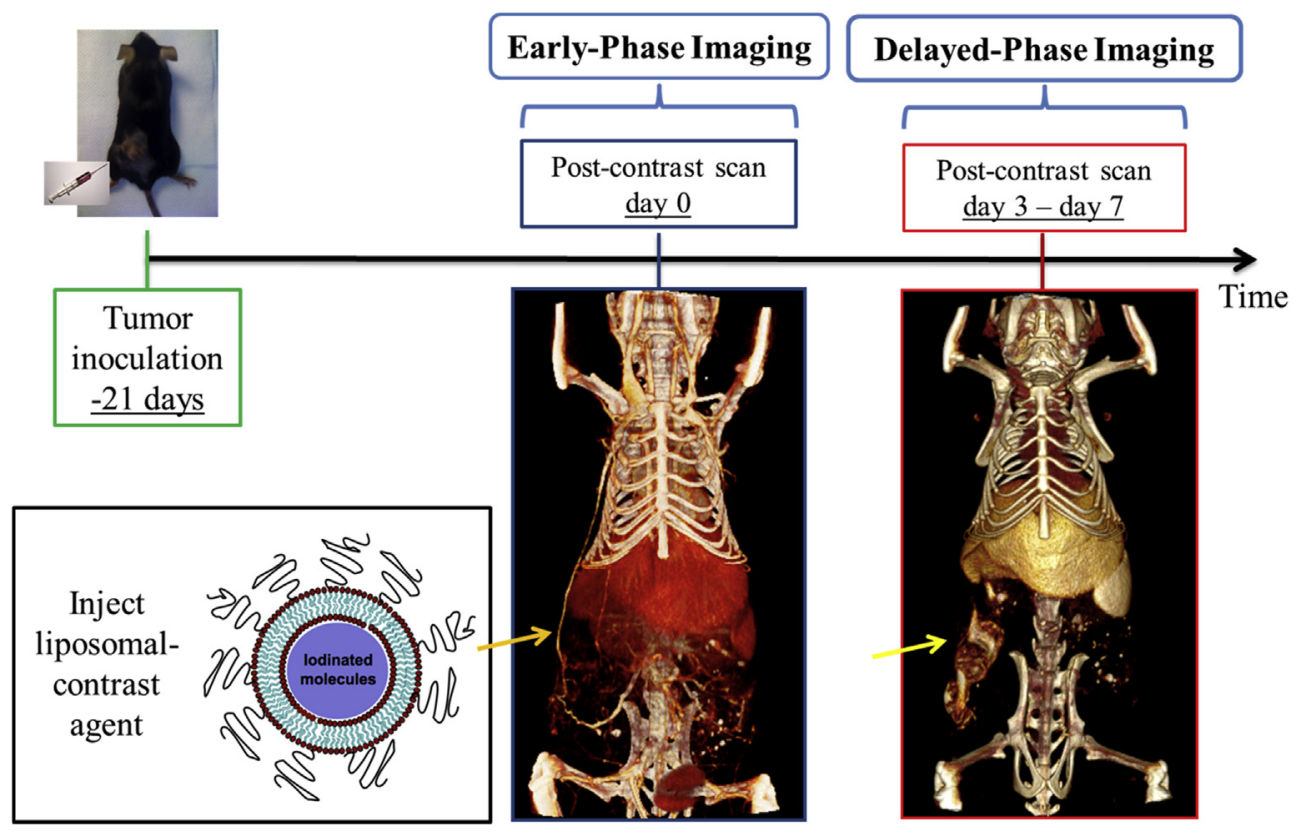

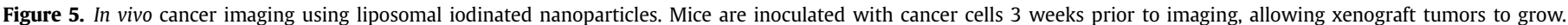

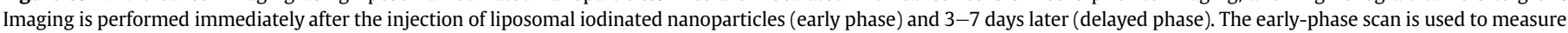
tumor fractional blood volume, while the delayed-phase scan is used to measure tumor vessel permeability via the enhanced permeability and retention effect.

simultaneous or sequential data acquisition [146-148]. Even in the case of hardware fusion, a rigid registration must be applied to match the reference frames of the different acquisition systems [149].

Micro-CT images are used in hybrid small animal micro-PET/CT scanners to perform the attenuation correction of micro-PET images. The data processing workflow for micro-CT attenuation correction (AC) is very similar to that used for correction of PET data from CT images in combined clinical PET/CT scanners. For this purpose, the CT voxel values are first scaled to the corresponding attenuation coefficient of $511 \mathrm{keV}$ gamma photons using a bilinear function [150]; then, the micro-CT volume is blurred with a 3D Gaussian kernel to match the resolution of the micro-PET image. Finally, the blurred attenuation-map is forward projected and the exponential is applied to generate the attenuation correction factors (ACF). The ACF are then applied to the original PET sinograms by element-wise multiplication.

Less straightforward is the attenuation correction of microSPECT or pinhole-SPECT data. For SPECT, attenuation depends on the actual location where the radioactive decay takes place, information that is not available in the projection space of the emission data. For this reason, the most used method for AC in SPECT simplifies the problem by considering a uniform attenuation coefficient through the entire object, thus avoiding the use of transmission data [151]. More accurate correction can be obtained by including the information of the micro-CT derived, coregistered, AC map into the system matrix and using a maximum-likelihood reconstruction algorithm [152,153].

The use of multiple pinhole collimators has resulted in microSPECT systems exhibiting sub-millimeter resolution, which are well suited for in vivo studies using rats and mice [154]. It is typically advantageous to combine such a micro-SPECT system with micro-CT, in order to provide high-resolution co-registered images of anatomy $[155,156]$.

Figure 6 illustrates the capability to perform co-registered lung imaging with micro-SPECT and micro-CT in a mouse model of pulmonary airway obstruction using the U-SPECT-II/CT system
(MILabs, Utrecht, The Netherlands) [157]. This hybrid system is fitted with a high-resolution, $0.35 \mathrm{~mm}$, multi-pinhole collimator. Prior to imaging, the anesthetized mouse was injected with $5 \mathrm{mCi}$ of Tc99m-MAA and a liposomal iodine contrast agent $(0.008 \mathrm{ml}$ per gram mouse) via a tail vein catheter. The micro-CT image provides the structural information required to understand the cause of this perfusion defect (i.e. the obstruction bead). Together micro-SPECT/ micro-CT images show how a region distal to the airway obstruction maintains a high air fraction consistent with air trapping and limited perfusion.

\section{Future directions}

\section{Spectral micro-CT}

As previously stated (Section Contrast agents), one of the major challenges for CT imaging is its poor contrast sensitivity. Increased contrast discrimination can be achieved using spectral CT combined with intrinsic (e.g. bone) and extrinsic (e.g. blood pool contrast agents) sources of contrast. Dual energy (DE) CT imaging using an X-ray energy integrating detector and a polychromatic Xray source is the simplest form of spectral CT [158]. Additional metallic beam filters placed between the source and the specimen (e.g. 1-2 mm of aluminum or copper) can be used to preferentially remove low energy photons and to improve spectral separation between polychromatic scans [159]. As an example, dual energy CT can be used to separate extrinsic iodine contrast from intrinsic calcium contrast by exploiting the energy dependence of iodine attenuation observed in a low energy scan (mean energy falls below the k-edge of iodine, $33.2 \mathrm{keV}$ ) and a filtered, higher energy scan (mean energy falls above the k-edge of iodine).

Despite its numerous applications in clinical practice, the translation of dual energy CT imaging to preclinical systems has been challenging. The main difference with clinical $\mathrm{CT}$ is that micro-CT images are inherently prone to a higher amount of noise as the spatial resolution is also much higher. Apart from the obvious sequential scanning at two different $\mathrm{kVps}$, DE micro-CT sampling 

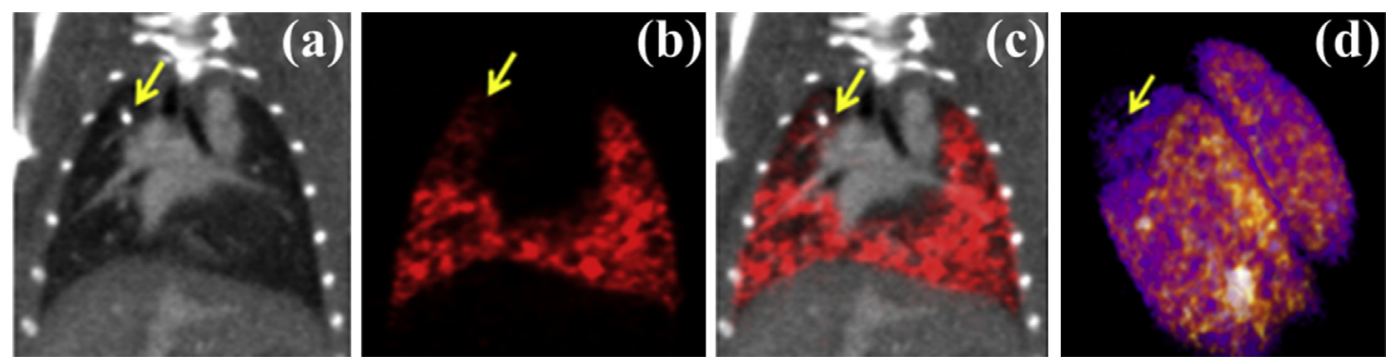

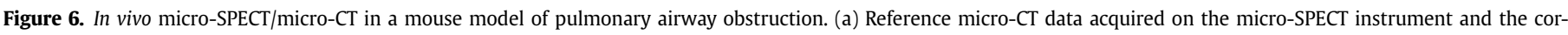

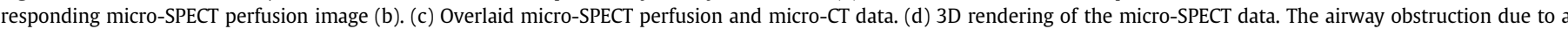

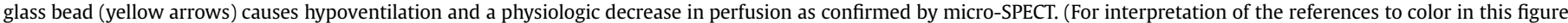
legend, the reader is referred to the web version of this article).

strategies include: 1) simultaneous dual source acquisition, and 2) single source acquisition with $\mathrm{kVp}$ switching. It has been found that $\mathrm{kVp}$ switching allows the most accurate results in DE decomposition, since it is not affected by cross-scattering as in dual source acquisitions [160]. Nominally, sequential and dual source scanning allow the use of a different beam filter for each $\mathrm{kVp}$; however, hardware based methods to allow filter switching in sync with $\mathrm{kVp}$ switching have been proposed and demonstrated [159].

Similar to the clinic, DE micro-CT data is used to perform material decomposition pre- or post-reconstruction (i.e. in the sinogram or image domain) [66]. Image domain decomposition has been preferred so far for micro-CT, given the ease of intensity normalization, image registration, and enhancement measurement in the image domain $[130,141,161-164]$. For each voxel, DE decomposition solves a system of equations with two non-negative unknowns representing the concentrations of two materials or two attenuation mechanisms (e.g. photoelectric effect and Compton scattering). Since noise is a major limiting factor in decomposition accuracy, applying image domain, joint bilateral filtration (BF; an adaptive, edge preserving smoothing filter) prior to DE decomposition was found to greatly improve the results of DE decomposition [165]. BF provided superior decomposition accuracy, precision, and elemental detectability relative to unfiltered data and to related filtration schemes which did not exploit spectral correlation in both phantom studies and in vivo data.

DE micro-CT has been used to separate 1 ) iodine and soft tissue in cardiac studies [130], 2) iodine and calcium in a study focused on imaging plaque-associated macrophages and in separating EPRbased accumulation of Lip-I in a tumor from bone [144,166], and $3)$ to separate iodine- and gold-based nanoparticles in tumors $[161,165]$. In the third case, DE micro-CT was used to measure both tumor vascular density and vessel permeability in soft-tissue sarcomas [165] and primary lung tumors [161]. Validation of the observed results was performed using histology and ex vivo measurement of tissue gold and iodine concentrations [161]. To illustrate, we show how two types of nanoparticle contrast agents based on gold $\mathrm{Au}$ ) and iodine (I) are used for vascular imaging with DE micro-CT of primary sarcoma tumors (Fig. 7). Injection of AuNPs followed by the injection of Lip-I 3 days later allowed simultaneous measurement of tumor fractional blood volume (FBV) using the iodine map and vascular permeability using the gold map. Scans acquired at 40 and $80 \mathrm{kVp}$ (with mean energies below and above the k-edge of iodine, respectively) were used to separate $\mathrm{Au}$ enhancement from I enhancement. Segmentation was required to separate bone from Au.

DE micro-CT and 4D cardiac micro-CT have been combined to provide 5D imaging [130] and applied in assessing cardiac injury in mice following partial-heart irradiation [164]. Radiation therapy applied in breast cancer can expose the heart to incidental radiation, which can lead to radiation-related heart disease and death. 4 and 8 weeks after 12 Gy partial heart irradiation, mice were injected with nanoparticle-based contrast agents and imaged with $\mathrm{DE}$ and $4 \mathrm{D}$ micro-CT to evaluate myocardial vascular permeability and cardiac function, respectively. Figure 8 displays DE results from this study, showing overlapped gold and iodine maps. The iodinated nanoparticles enabled 4D imaging for cardiac function estimation. AuNP accumulation in the injured myocardium resulted from the EPR effect at the site of radiation damage.

DE micro-CT can also be applied to separate three materials. A material separation algorithm, developed by Granton et al. [167] can be applied to separate, for example, bone, iodine, and soft tissue. The three material decomposition algorithm assumes that the volume fractions of each basis material in a voxel add to unity. A similar approach has been applied to separate air, iodine, and soft tissue in the lungs [163].

A promising alternative to $\mathrm{DE}$ micro-CT can be provided by PCXDs which acquire perfectly registered data over multiple energy bins with a single scan. The reconstruction process attempts to recover the attenuation coefficient for each specific energy bin (range) enabling high fidelity k-edge imaging with a polychromatic X-ray source and, ideally, emulating k-edge imaging results produced with a tunable, monochromatic, synchrotron source [123]. Paralleling the development of fluorescence optical imaging, a natural extension of these studies is to introduce multiple probes that incorporate different high- $Z$ materials (e.g. iodine, barium, gold, gadolinium) to allow co-localization studies using PCXDs [168].

Given the limited field-of-view covered by most current PCXDs, hybrid micro-CT systems have been designed that combine a traditional micro-CT imaging chain with a spectral imaging chain [169]. Such hybrid systems use interior tomography for spectral reconstruction within the PCXD's field of view with very promising results. Although many technical problems remain to be solved, it is largely agreed that PCXDs are the next step in CT imaging and that their adoption in the preclinical arena will ultimately translate to new clinical applications A PCXD-based mammography system consisting of a large number of crystalline silicon strip detectors has recently been introduced by Philips (the MicroDose system).

There is little doubt that spectral imaging and novel nanoparticle based contrast agents have the potential to significantly augment CT imaging. Furthermore, some of the CT nanoparticles such as AuNP can be used both for tumor detection since they accumulate in tumors via the EPR effect [142] and therapy. Using mice, Hainfeld et al. [170-172] have shown that tumors loaded with AuNP receive a higher radiation dose than normal tissue without AuNP during radiation treatment, recommending AuNP as possible radiosensitizers in cancer treatment. 


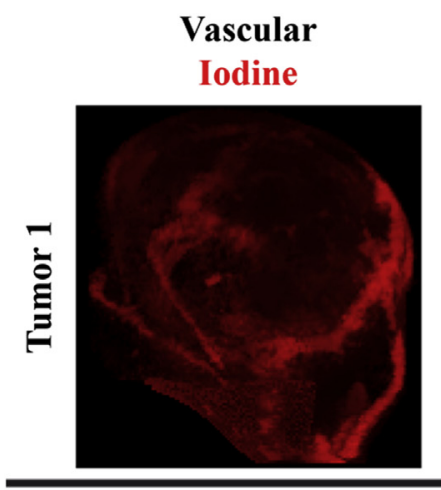

\section{Extravasated \\ Gold}
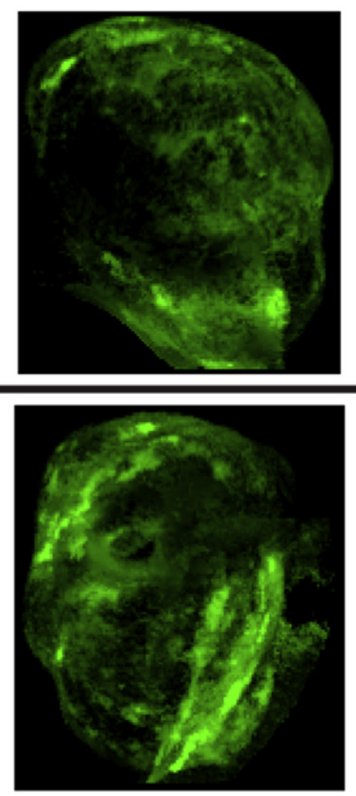

\section{Combined}
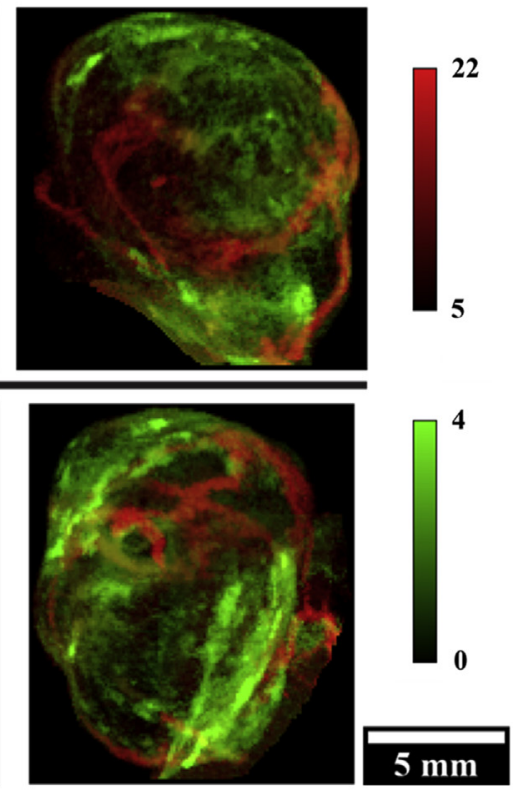

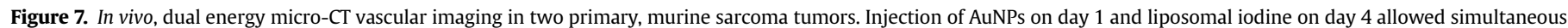

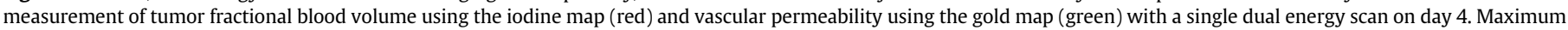

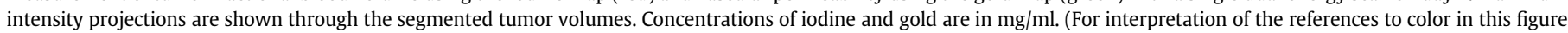
legend, the reader is referred to the web version of this article).
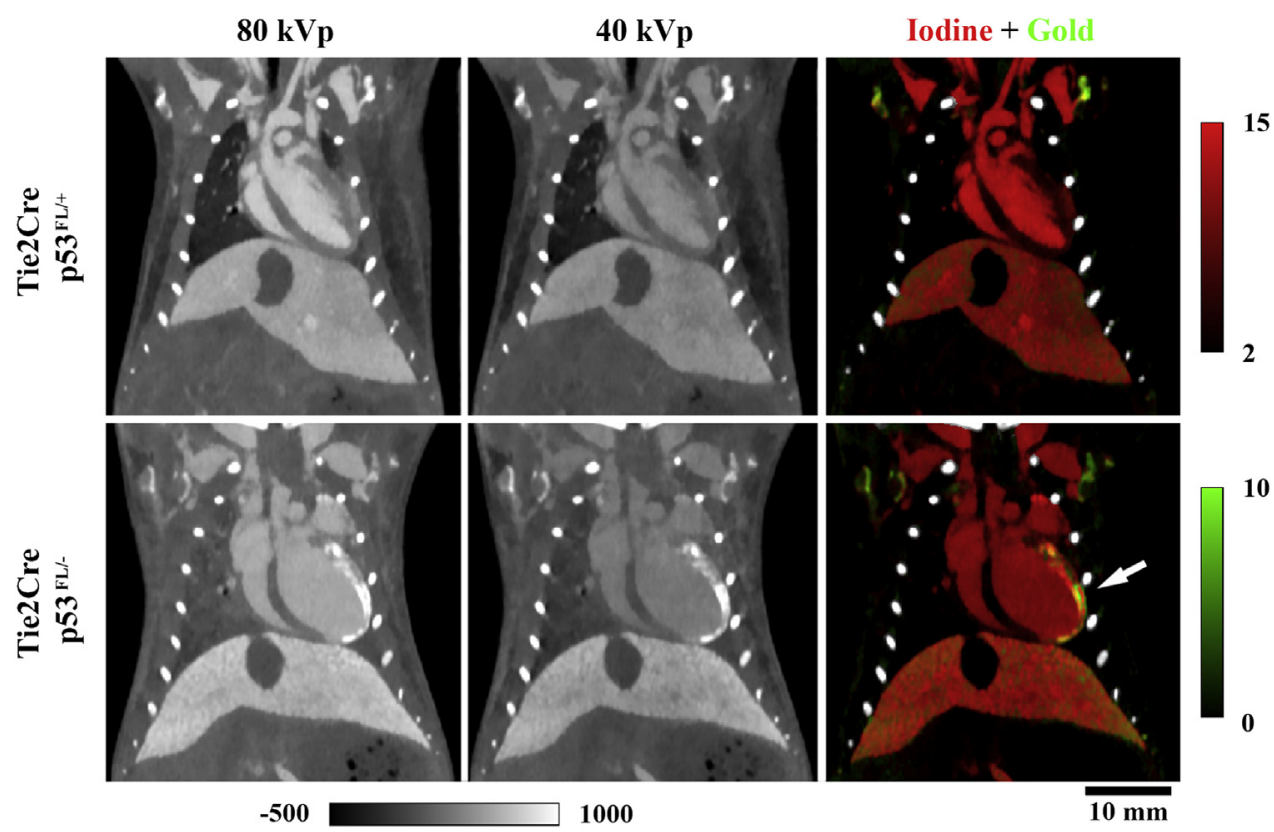

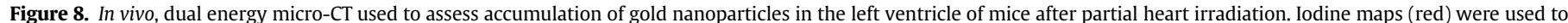

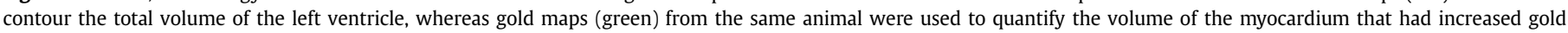

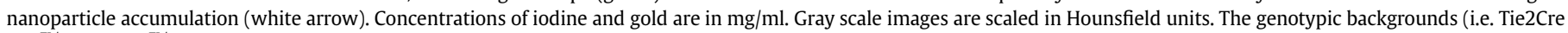
$\mathrm{p} 53^{\mathrm{FL} /-}$ and $\mathrm{p} 53^{\mathrm{FL} /+}$ ) are detailed in Ref. [164]. (For interpretation of the references to color in this figure legend, the reader is referred to the web version of this article).

\section{Phase contrast imaging}

A common objective of in vivo spectral CT is to separate contrast agents based on high atomic number materials $(Z>50)$ from biological soft tissues $(Z \leq 20)$ based on energy-dependent discontinuities in mass attenuation (k-edges) which occur within the diagnostic X-ray range ( $30-140 \mathrm{keV})$. For such applications, X-ray photons are treated as particles. Treating X-rays instead as electromagnetic waves, their complex index of refraction is expressed as $n=1-\delta-i \beta$ which relates to the linear absorption coefficient, $\mu_{0}$, as $\beta=\mu_{0}{ }^{*}(\lambda / 4 \pi)$ and to the coefficient of phase change, $\eta$, as $\delta=\eta^{*}(\lambda / 2 \pi)$ for X-ray wavelength, $\lambda$. For diagnostic X-rays 
A) Micro-CT, Synchrotron Source

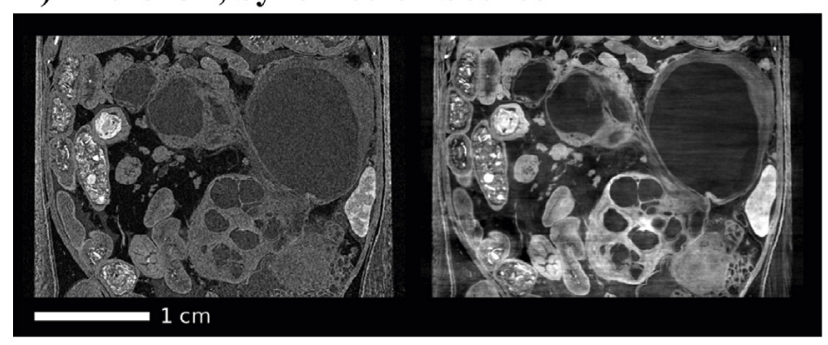

B) Micro-CT, Tube Source

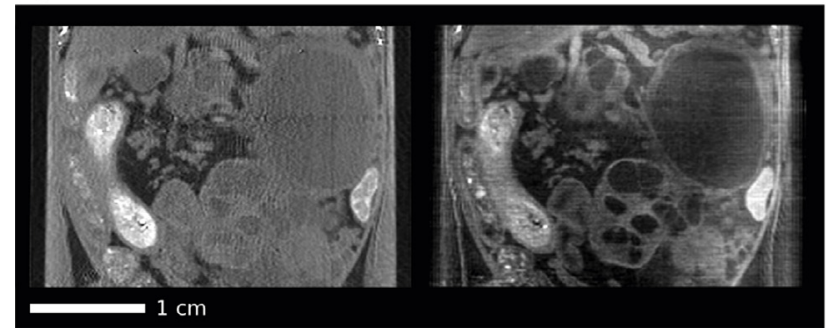

C) MRI

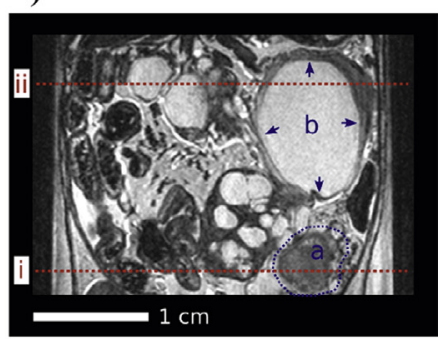

D) Histology

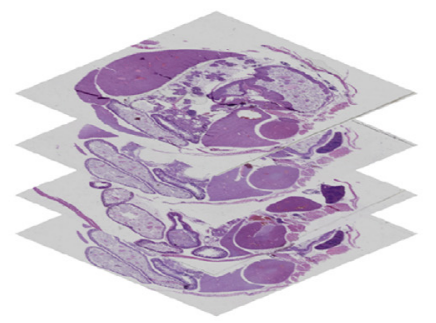

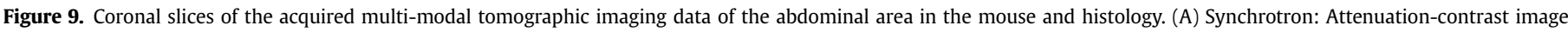

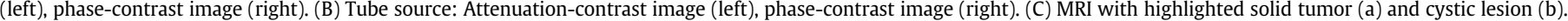

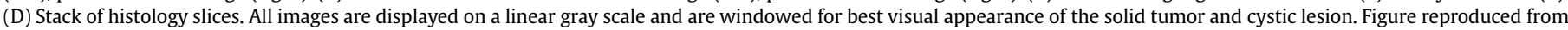
Ref. [181] with permission. All results shown in this figure were acquired ex vivo after whole-animal perfusion fixation with paraformaldehyde.

(10-100 keV) and low atomic number elements $(Z \leq 20), \delta$ is $\sim 2-3$ orders of magnitude larger than $\beta$ and falls off more slowly with increasing X-ray energy $\left(\sim 1 / \mathrm{E}^{2}\right.$ vs. $\left.\sim 1 / \mathrm{E}^{3}\right)$. Because of these factors, phase contrast is lauded for its potential in imaging soft biological tissues without the need for exogenous contrast agents and with potentially reduced radiation dose $[59,173]$.

These theoretical advantages have been practically validated using monochromatic, synchrotron X-ray sources and one of several imaging schemes, including (1) propagation based, (2) analyzer-crystal based, and (3) grating interferometer based phase contrast. Propagation based phase contrast imaging relies on the propagation distance of X-rays after they have been refracted within the sample (i.e. on the object-detector distance). Rotating the sample and acquiring projections at a single distance allows reconstruction of hybrid, edge-enhanced data in which the absorption information is augmented by the Laplacian of the refractive index. Explicit phase and absorption retrieval are possible by acquiring the projections at several object-detector distances followed by algorithmic post-processing [174]. Analyzer crystal based methods rely on Bragg diffraction within a silicon single crystal. Acquiring projections with a fixed sample position as the angle of the analyzer crystal is varied with respect to the X-ray beam exiting the sample yields a "rocking curve" (crystal angle vs. recorded intensity) from which refraction and apparent absorption projections can be recovered [175]. Grating interferometer based phase contrast methods use aligned, micron-scale gratings to generate systematic interference fringes in the propagating X-ray wavefront. Refraction within the sample shifts the interference fringes, allowing recovery of the differential of phase shifts caused by the object from intensity curves generated by stepping the sample or a component grating through a grating period (phase stepping) while the sample position remains fixed [176].

One of the primary challenges in translating these phase contrast imaging methods from synchrotron beam lines to routine clinical and preclinical application is the comparatively low brilliance and low coherence of laboratory X-ray sources; however, technological developments is this area are very promising. Pfeiffer et al. have demonstrated the effective translation of the Talbot-Lau grating interferometer from a synchrotron source to a polychromatic X-ray tube source through the addition of a source grating which collimates the source beam into an array of individually coherent X-ray lines sources [177]. They have also demonstrated the recovery of three contrast mechanisms with a single scan (absorption contrast, differential phase contrast, and dark field scatter contrast) $[178,179]$ and have developed a prototype small animal scanner in collaboration with Bruker MicroCT $[179,180]$. Additionally, they have demonstrated the potential value of differential phase contrast and dark field imaging in several applications including cancer identification and classification [181,182] and lung imaging [183]. Figure 9 is adapted [181] and compares phase and absorption contrast results using a synchrotron and laboratory source with magnetic resonance imaging (MRI) data and histology in a mouse model of pancreatic ductal adenocarcinoma. As an alternative to the Talbot-Lau interferometer with source grating, a non-interferometric, grating based approach for differential phase contrast with a laboratory source, called coded aperture X-ray phase contrast imaging, has been proposed and demonstrated using a physical phantom [184]. Additional work has been done with an analyzer crystal based setup and a laboratory source [185]. A commercial mammography scanner which exploits propagation based edge enhancement has been developed by Konica Minolta, Inc (the REGIUS PureView).

Several technical challenges must be overcome prior to the wide-spread adoption of phase contrast imaging. High quality tomographic reconstructions of differential phase contrast data using a Talbot-Lau grating interferometer can require hours to tens of hours of scanning owing to the flux limitations associated with laboratory X-ray sources which are exacerbated by collimator-like phase gratings and owing to the phase stepping performed at each sample rotation angle [186]. A single-shot alternative to phase stepping which uses Moiré patterns and Fourier analysis has been demonstrated using a physical phantom and could lead to significantly reduced scan times [187]. Phase wrapping in thick specimens and bone poses a significant hurdle for preclinical and clinical imaging; however, algorithms have already been proposed and demonstrated for phase unwrapping [180,188]. Additional 
fabrication challenges are associated with scaling the field-of-view and viable energy range for grating interferometers. Proposed solutions to these problems include helical scanning [186] and inclining the gratings with respect to the beam direction for reduced transmittance of high energy photons [189], respectively.

In addition to technological developments, the adaptation of iterative reconstruction algorithms to the problem of tomographic phase-contrast image reconstruction will undoubtly reduce associated scanning times and radiation dose. Preliminary work has been presented in literature illustrating how existing iterative CT reconstruction algorithms can be modified for this purpose [190,191]. Furthermore, techniques for enforcing gradient sparsity in phase contrast data have been demonstrated [192,193].

\section{Conclusions}

Micro-CT provides a reliable platform for small animal imaging that is complementary to other small animal imaging methods, enabling numerous morphological and functional imaging applications. The radiation dose and low contrast associated with X-ray imaging are well-known; however, newly developed contrast agents and novel acquisition and reconstruction strategies show extraordinary promise in overcoming these limitations. Combined with exciting new opportunities in spectral and phase contrast imaging, these developments will surely continue to expand the applications for micro-CT in small animal models.

\section{Acknowledgments}

Some of the work presented here was performed at the Duke Center for In Vivo Microscopy, an NIH/NIBIB national Biomedical Technology Resource Center (P41 EB015897). Liposomal contrast agent was provided by Ketan Ghaghada and Ananth Annapragrada (Texas Children's Hospital, Houston, TX). We thank Sally Zimney for the editorial assistance, and Drs. G. Allan Johnson and Nicholas Befera for their contributions related to micro-SPECT.

\section{References}

[1] Holdsworth DW, Thornton M. Micro-CT in small animal and specimen imaging. Trends Biotechnol 2002;20(8):S34-39.

[2] Feldkamp LA, Davis LC, Kress JW. Practical cone-beam algorithm. J Opt Soc Am 1984;1(6):612-9.

[3] Johnston SM, Johnson GA, Badea CT. Geometric calibration for a dual tube detector micro-CT system. Med Phys 2008;35(5):1820-9.

[4] Badea CT, Hedlund LW, Johnson GA. Micro-CT with respiratory and cardiac gating. Med Phys 2004;31(12):3324-9.

[5] Badea CT, Schreibmann E, Fox T. A registration-based approach for cardiac micro-CT using combined prospective and retrospective gating. Med Phys 2008;35(4):1170-9.

[6] Cao G, Burk LM, Lee YZ, Calderon-Colon X, Sultana S, Lu J, et al. Prospectivegated cardiac micro-CT imaging of free-breathing mice using carbon nanotube field emission x-ray. Med Phys 2010;37(10):5306-12.

[7] Cao G, Lee YZ, Peng R, Liu Z, Rajaram R, Calderon-Colon X, et al. A dynamic micro-CT scanner based on a carbon nanotube field emission $\mathrm{X}$-ray source. Phys Med Biology 2009;54(8):2323.

[8] Yue G, Qiu Q, Gao B, Cheng Y, Zhang J, Shimoda H, et al. Generation of continuous and pulsed diagnostic imaging $\mathrm{X}$-ray radiation using a carbonnanotube-based field-emission cathode. Appl Phys Lett 2002;81(2):355-7.

[9] Grider DE, Wright A, Ausburn PK. Electron beam melting in microfocus X-ray tubes. J Phys D Appl Phys 1986;19(12):2281.

[10] Fivez CM, Wambacq P, Suetens P, Schoeters EP. Influence of heel effect and of nonuniformity of emitted spectra on dual-energy subtraction in computed radiography. Proc SPIE 1996;2708:588-98.

[11] Brunke O, Brockdorf K, Drews S, Müller B, Donath T, Herzen J, et al. Comparison between $\mathrm{X}$-ray tube-based and synchrotron radiation-based $\mu \mathrm{CT}$; 2008. p. 70780U-70780U.

[12] Ritman EL, Jorgensen SM, Beighley PE, Thomas PJ, Dunsmuir JH, Romero JC et al. Synchrotron-based micro-CT of in-situ biological basic functional units and their integration; 1997. pp. 13-24.

[13] Stolz E, Yeniguen M, Kreisel M, Kampschulte M, Doenges S, Sedding D, et al Angioarchitectural changes in subacute cerebral venous thrombosis. A synchrotron-based micro- and nano-CT study. NeuroImage 2011;54(3):1881-6.
[14] Kujoory MA, Hillman BJ, Barrett HH. High-resolution computed tomography of the normal rat nephrogram. Invest Radiol 1980;15(2):148-54.

[15] Feldkamp LA, Goldstein SA, Parfitt AM, Jesion G, Kleerekoper M. The direct examination of three-dimensional bone architecture in vitro by computed tomography. J Bone Miner Res 1989;4(1):3-11.

[16] Holdsworth DW, Drangova M, Fenster A. A high-resolution XRII-based quantitative volume CT scanner. Med Phys 1993;20(2 Pt 1):449-62.

[17] Boone JM, Alexander GM, Seibert JA. A fluoroscopy-based computed tomography scanner for small specimen research. Invest Radiol 1993;28(6): 539-44.

[18] Paulus MJ, Gleason SS, Kennel SJ, Hunsicker PR, Johnson DK. High resolution $\mathrm{X}$-ray computed tomography: an emerging tool for small animal cancer research. Neoplasia 2000;2(1-2):62-70.

[19] Goertzen AL, Nagarkar V, Street RA, Paulus MJ, Boone JM, Cherry SR. A comparison of $\mathrm{X}$-ray detectors for mouse CT imaging. Phys Med Biol 2004;49(23):5251-65.

[20] Lee SC, Kim HK, Chun IK, Cho MH, Lee SY, Cho MH. A flat-panel detector based micro-CT system: performance evaluation for small-animal imaging. Phys Med Biol 2003;48(24):4173-85.

[21] Kalender WA, Kyriakou Y. Flat-detector computed tomography (FD-CT). Eur Radiol 2007;17(11):2767-79.

[22] Panetta D, Belcari N, Baldazzi G, Carpentieri C, Cicalini E, Del Guerra A, et al. Characterization of a high-resolution CT scanner prototype for small animals. Nuovo Cimento Della Societa Italiana Di Fisica B-General Physics Relativity Astronomy and Mathematical Physics and Methods 2007;122(6-7):739-47.

[23] Roessl E, Cormode D, Brendel B, Jürgen Engel K, Martens G, Thran A, et al. Preclinical spectral computed tomography of gold nano-particles. Nucl Instruments Methods Phys Res Sect Accel Spectrom Detect Assoc Equip 2011;648(Supplement 1(0)):S259-64.

[24] Taguchi K, Iwanczyk JS. Vision 20/20: single photon counting X-ray detectors in medical imaging. Med Phys 2013;40(10):100901.

[25] Taguchi K, Xu J, Srivastava S, Tsui BM, Cammin J, Tang Q. Interior region-ofinterest reconstruction using a small, nearly piecewise constant subregion. Med Phys 2011:38(3):1307-12.

[26] Bornefalk H, Danielsson M. Photon-counting spectral computed tomography using silicon strip detectors: a feasibility study. Phys Med Biol 2010;55(7): 1999-2022.

[27] Yu H, Xu Q, He P, Bennett J, Amir R, Dobbs B, et al. Medipix-based Spectral Micro-CT. CT Li Lun Yu Ying Yong Yan Jiu 2012;21(4):583.

[28] Campbell M, Heijne E, Meddeler G, Pernigotti E, Snoeys W. A readout chip for a $64 \times 64$ pixel matrix with 15-bit single photon counting. Nucl Sci IEEE Transactions 1998;45(3):751-3.

[29] Badea C, Drangova M, Holdsworth D, Johnson G. In vivo small-animal imaging using micro-CT and digital subtraction angiography. Phys Med Biol 2008;53:R319.

[30] Drangova M, Ford NL, Detombe SA, Wheatley AR, Holdsworth DW. Fast Retrospectively gated quantitative four-dimensional (4D) cardiac micro computed tomography imaging of free-breathing mice. Invest Radiol 2007;42(2):85-94.

[31] Bartling SH, Stiller W, Grasruck M, Schmidt B, Peschke P, Semmler W, et al. Retrospective motion gating in small animal CT of mice and rats. Invest Radiol 2007;42(10):704-14.

[32] Badea CT, Fubara B, Hedlund LW, Johnson GA. 4-D micro-CT of the mouse heart. Mol Imaging 2005;4(2):110-6.

[33] Panetta D, Belcari N, Del Guerra A, Bartolomei A, Salvadori P. Analysis of image sharpness reproducibility on a novel engineered micro-CT scanner with variable geometry and embedded recalibration software. Physica Medica 2012;28(2):166-73.

[34] Panetta D, Belcari N, Del Guerra A, Moehrs S. An optimization-based method for geometrical calibration in cone-beam CT without dedicated phantoms. Phys Med Biol 2008;53(14):3841-61.

[35] Wicklein J, Kunze H, Kalender WA, Kyriakou Y. Image features for misalignment correction in medical flat-detector CT. Med Phys 2012;39(8): 4918-31.

[36] Paulus MJ, Gleason SS, Easterly ME, Foltz CJ. A review of high-resolution Xray computed tomography and other imaging modalities for small animal research. Lab Anim (NY) 2001;30(3):36-45.

[37] Kak AC, Slaney M. Principles of computerized tomographic imaging. New York: IEEE Press; 1988.

[38] Tuy H. An inversion formula for cone-beam reconstruction. SIAM J Appl Math $1983 ; 43: 546-62$.

[39] Defrise M, Clack R. Filtered backprojection reconstruction of combined parallel beam and cone beam SPECT data. Phys Med Biol 1995:40(9):1517-37.

[40] Gordon R, Bender R, Herman GT. Algebraic reconstruction techniques (ART) for three-dimensional electron microscopy and x-ray photography. J Theor Biol 1970:29:471-81.

[41] Rudin LI, Osher S, Fatemi E. Nonlinear total variation based noise removal algorithms. Physica D 1992;60:259-68.

[42] Song J, Liu QH, Johnson GA, Badea CT. Sparseness prior based iterative image reconstruction for retrospectively gated cardiac micro-CT. Med Phys 2007;34(11):4476-83.

[43] Elbakri IA, Fessler JA. Statistical image reconstruction for polyenergetic X-ray computed tomography. IEEE Trans Med Imaging 2002;21:89-99.

[44] Xu F, Mueller K. Real-time 3D computed tomographic reconstruction using commodity graphics hardware. Phys Med Biol 2007;52(12):3405-19. 
[45] Jakab G, Racz A, Major P, Buekki T, Nemeth G. Fully GPU based real time corrections and reconstruction for cone beam micro CT. In: Yu B, editor. 2009 Ieee Nuclear Science Symposium Conference Record, vol. 1-5; 2009. pp. 4068-71.

[46] Johnston SM, Johnson GA, Badea CT. GPU-based iterative reconstruction with total variation minimization for micro-CT. Proc. SPIE 2010:7622:762238.

[47] Zhao X, Bian J, Sidky EY, Cho S, Zhang P, Pan X, et al. GPU-based 3D conebeam CT image reconstruction: application to micro CT. In: 2007 leee Nuclear Science Symposium Conference Record, vol. 1-11; 2007. pp. 3922-5.

[48] Guo XL, Johnston SM, Johnson GA, Badea CT. A comparison of sampling strategies for dual energy micro-CT. Medical imaging 2012: physics of medical imaging 2012:8313.

[49] Kachelriess M, Sennst DA, Maxlmoser W, Kalender WA. Kymogram detection and kymogram-correlated image reconstruction from subsecond spiral computed tomography scans of the heart. Med Phys 2002;29(7):1489-503.

[50] Dinkel J, Bartling SH, Kuntz J, Grasruck M, Kopp-Schneider A, Iwasaki M, et al. Intrinsic gating for small-animal computed tomography a robust ECG-less paradigm for deriving cardiac phase information and functional imaging. Circulation-Cardiovascular Imaging 2008;1(3):235-43.

[51] Bartling SH, Dinkel J, Stiller W, Grasruck M, Madisch I, Kauczor HU, et al. Intrinsic respiratory gating in small-animal CT. Eur Radiol 2008;18(7): 1375-84.

[52] Sawall S, Bergner F, Lapp R, Mronz M, Karolczak M, Hess A, et al. Low-dose cardio-respiratory phase-correlated cone-beam micro-CT of small animals. Med Phys 2011;38(3):1416-24.

[53] Johnson JT, Hansen MS, Wu I, Healy LJ, Johnson CR, Jones GM, et al. Virtual histology of transgenic mouse embryos for high-throughput phenotyping. PLoS Genet 2006;2(4):e61.

[54] Metscher BD. MicroCT for developmental biology: a versatile tool for highcontrast 3D imaging at histological resolutions. Dev Dyn 2009;238(3): $632-40$.

[55] Metscher BD. MicroCT for comparative morphology: simple staining methods allow high-contrast 3D imaging of diverse non-mineralized animal tissues. BMC Physiol 2009;9:11.

[56] Lin MD, Ning L, Badea CT, Mistry NN, Qi Y, Johnson GA. A high precision contrast injector for small animal $\mathrm{X}$-ray digital subtraction angiography. IEEE Trans Biomed Eng 2008;2008(3):1082-91.

[57] Ashton JR, Befera N, Clark D, Qi Y, Mao L, Rockman HA, et al. Anatomical and functional imaging of myocardial infarction in mice using micro-CT and eXIA 160 contrast agent. Contrast Media Mol Imaging 2014;9(2):161-8.

[58] Mukundan Jr S, Ghaghada KB, Badea CT, Kao CY, Hedlund LW, Provenzale JM, et al. A liposomal nanoscale contrast agent for preclinical CT in mice. AJR Am J Roentgenol 2006;186(2):300-7.

[59] Momose A. Recent advances in X-ray phase imaging. Jpn J Appl Phys 2005;44(9R):6355.

[60] Popovtzer R, Agrawal A, Kotov NA, Popovtzer A, Balter J, Carey TE, et al. Targeted gold nanoparticles enable molecular CT imaging of cancer. Nano Lett 2008:8(12):4593-6.

[61] Reuveni E. The genetic background effect on domesticated species: a mouse evolutionary perspective. ScientificWorld Journal 2011;11:429-36.

[62] Wyss C, Schaefer SC, Juillerat-Jeanneret L, Lagopoulos L, Lehr HA, Becker CD et al. Molecular imaging by micro-CT: specific E-selectin imaging. Eur Radiol 2009;19(10):2487-94.

[63] Ghann WE, Aras O, Fleiter T, Daniel MC. Syntheses and characterization of lisinopril-coated gold nanoparticles as highly stable targeted CT contrast agents in cardiovascular diseases. Langmuir 2012;28(28):10398-408.

[64] Chanda N, Shukla R, Zambre A, Mekapothula S, Kulkarni RR, Katti K, et al. An effective strategy for the synthesis of biocompatible gold nanoparticles using cinnamon phytochemicals for phantom CT imaging and photoacoustic detection of cancerous cells. Pharm Res 2011;28(2):279-91.

[65] Cormode DP, Roessl E, Thran A, Skajaa T, Gordon RE, Schlomka JP, et al. Atherosclerotic plaque composition: analysis with multicolor CT and targeted gold nanoparticles. Radiology 2010;256(3):774-82.

[66] Schirra CO, Brendel B, Anastasio MA, Roessl E. Spectral CT: a technology primer for contrast agent development. Contrast Media Mol Imaging 2014;9(1):62-70.

[67] Winter PM, Shukla HP, Caruthers SD, Scott MJ, Fuhrhop RW, Robertson JD, et al. Molecular imaging of human thrombus with computed tomography, Acad Radiol 2005:12(5):S9-13.

[68] Ford NL, Thornton MM, Holdsworth DW. Fundamental image quality limits for microcomputed tomography in small animals. Med Phys 2003;30: 2869-77.

[69] Boone JM, Velazquez O, Cherry SR. Small-animal X-ray dose from micro-CT. Mol Imaging 2004;3(3):149-58.

[70] Taschereau R, Chow PL, Chatziioannou AF. Monte carlo simulations of dose from microCT imaging procedures in a realistic mouse phantom. Med Phys 2006;33(1):216-24.

[71] Beckmann N, Kneuer R, Gremlich HU, Karmouty-Quintana H, Ble FX, Muller M. In vivo mouse imaging and spectroscopy in drug discovery. NMR Biomed 2007;20(3):154-85.

[72] Kohn HI, Kallman RF. The influence of strain on acute x-ray lethality in the mouse. II. Recovery rate studies. Radiat Res 1957;6(3):329-38.

[73] Sato F, Sasaki S, Kawashima N, Chino F. Late effects of whole or partial body $\mathrm{x}$-irradiation on mice: life shortening. Int J Radiat Biol Relat Stud Phys Chem Med 1981;39(6):607-15.
[74] Bond VP, Robertson JS. Vertebrate radiobiology (lethal actions and associated effects). Annu Rev Nucl Sci 1957;7:135-62.

[75] Mole RH. Quantitative observations on recovery from whole body irradiation in mice. II. Recovery during and after daily irradiation. $\mathrm{Br} \mathrm{J}$ Radiol 1957;30(349):40-6.

[76] Carlson SK, Classic KL, Bender CE, Russell SJ. Small animal absorbed radiation dose from serial micro-computed tomography imaging. Mol Imaging Biol 2007;9(2):78-82.

[77] Lehnert S, Rybka WB. Dose rate dependence of response of mouse lung to irradiation. Br J Radiol 1985;58(692):745-9.

[78] Parkins CS, Fowler JF, Maughan RL, Roper MJ. Repair in mouse lung for up to 20 fractions of X rays or neutrons. Br J Radiol 1985;58(687):225-41.

[79] Detombe SA, Dunmore-Buyze J, Petrov IE, Drangova M. X-ray dose delivered during a longitudinal micro-CT study has no adverse effect on cardiac and pulmonary tissue in C57BL/6 mice. Acta Radiologica 2013;54(4):435-41.

[80] Kinney JH, Lane NE, Haupt DL. In vivo, three-dimensional microscopy of trabecular bone. J Bone Miner Res 1995;10(2):264-70.

[81] Ruegsegger P, Koller B, Muller R. A microtomographic system for the nondestructive evaluation of bone architecture. Calcif Tissue Int 1996;58(1): 24-9.

[82] Chappard C, Basillais A, Benhamou L, Bonassie A, Brunet-Imbault B, Bonnet N, et al. Comparison of synchrotron radiation and conventional X-ray microcomputed tomography for assessing trabecular bone microarchitecture of human femoral heads. Med Phys 2006;33(9):3568-77.

[83] Appleton CT, McErlain DD, Pitelka V, Schwartz N, Bernier SM, Henry JL, et al Forced mobilization accelerates pathogenesis: characterization of a preclinical surgical model of osteoarthritis. Arthritis Res Ther 2007:9(1):R13.

[84] McErlain DD, Appleton CT, Litchfield RB, Pitelka V, Henry JL, Bernier SM, et al Study of subchondral bone adaptations in a rodent surgical model of OA using in vivo micro-computed tomography. Osteoarthr Cartil 2008;16(4): 458-69.

[85] Cowan CM, Aghaloo T, Chou YF, Walder B, Zhang X, Soo C, et al. MicroCT evaluation of three-dimensional mineralization in response to BMP-2 doses in vitro and in critical sized rat calvarial defects. Tissue Eng 2007;13(3): $501-12$.

[86] Waarsing JH, Day JS, Weinans H. Longitudinal micro-CT scans to evaluate bone architecture. J Musculoskelet Neuronal Interact 2005;5(4):310-2.

[87] David V, Laroche N, Boudignon B, Lafage-Proust $\mathrm{MH}$, Alexandre C, Ruegsegger $\mathrm{P}$, et al. Noninvasive in vivo monitoring of bone architecture alterations in hindlimb-unloaded female rats using novel three-dimensional microcomputed tomography. J Bone Miner Res 2003;18(9):1622-31.

[88] Bouxsein ML, Boyd SK, Christiansen BA, Guldberg RE, Jepsen KJ, Muller R. Guidelines for assessment of bone microstructure in rodents using microcomputed tomography. J Bone Miner Res 2010;25(7):1468-86.

[89] Li R, Stewart DJ, Von Schroeder HP, Mackinnon ES, Schemitsch EH. Effect of cell-based VEGF gene therapy on healing of a segmental bone defect. J Orthop Res 2009;27(1):8-14.

[90] Lee SW, Padmanabhan P, Ray P, Gambhir SS, Doyle T, Contag C, et al. Stem cell-mediated accelerated bone healing observed with in vivo molecular and small animal imaging technologies in a model of skeletal injury. J Orthop Res 2009;27(3):295-302.

[91] Dumas A, Brigitte M, Moreau MF, Chrétien F, Baslé MF, Chappard D. Bone mass and microarchitecture of irradiated and bone marrow-transplanted mice: influences of the donor strain. Osteoporos Int 2009;20(3):435-43.

[92] Laib A, Kumer JL, Majumdar S, Lane NE. The temporal changes of trabecular architecture in ovariectomized rats assessed by microCT. Osteoporos Int $2001 ; 12(11): 936-41$

[93] Laib A, Barou O, Vico L, Lafage-Proust MH, Alexandre C, Rugsegger P. 3D micro-computed tomography of trabecular and cortical bone architecture with application to a rat model of immobilisation osteoporosis. Med Biol Eng Comput 2000;38(3):326-32.

[94] Dempster DW, Cosman F, Kurland ES, Zhou H, Nieves J, Woelfert L, et al Effects of daily treatment with parathyroid hormone on bone microarchitecture and turnover in patients with osteoporosis: a paired biopsy study. J Bone Miner Res 2001;16(10):1846-53.

[95] Umoh JU, Sampaio AV, Welch I, Pitelka V, Goldberg HA, Underhill TM, et al. In vivo micro-CT analysis of bone remodeling in a rat calvarial defect model. Phys Med Biol 2009;54(7):2147-61.

[96] Lin CY, Schek RM, Mistry AS, Shi X, Mikos AG, Krebsbach PH, et al. Functional bone engineering using ex vivo gene therapy and topology-optimized, biodegradable polymer composite scaffolds. Tissue Eng 2005;11(9-10) 1589-98.

[97] Ho ST, Hutmacher DW. A comparison of micro CT with other techniques used in the characterization of scaffolds. Biomaterials 2006;27(8):1362-76.

[98] Borah B, Gross GJ, Dufresne TE, Smith TS, Cockman MD, Chmielewski PA, et al. Three-dimensional microimaging (MR $\mathrm{I}$ and $\mu \mathrm{CT}$ ), finite element modeling, and rapid prototyping provide unique insights into bone architecture in osteoporosis. Anat Rec 2001;265(2):101-10.

[99] Guldberg RE, Lin AS, Coleman R, Robertson G, Duvall C. Microcomputed tomography imaging of skeletal development and growth. Birth Defects Res C Embryo Today 2004;72(3):250-9.

[100] Bartling SH, Stiller W, Semmler W, Kiessling F. Small animal computed tomography imaging. Curr Med Imaging Rev 2007:3(1):45-59.

[101] Missbach-Guentner J, Dullin C, Zientkowska M, Domeyer-Missbach M Kimmina S, Obenauer S, et al. Flat-panel detector-based volume computed 
tomography: a novel 3D imaging technique to monitor osteolytic bone lesions in a mouse tumor metastasis model. Neoplasia 2007;9(9):755-65.

[102] Martin-Badosa E, Amblard D, Nuzzo S, Elmoutaouakkil A, Vico L, Peyrin F. Excised bone structures in mice: imaging at three-dimensional synchrotron radiation micro CT. Radiology 2003;229(3):921-8.

[103] Ding M, Odgaard A, Hvid I. Accuracy of cancellous bone volume fraction measured by micro-CT scanning. J Biomechanics 1999;32(3):323-6.

[104] Buie HR, Campbell GM, Klinck RJ, MacNeil JA, Boyd SK. Automatic segmentation of cortical and trabecular compartments based on a dual threshold technique for in vivo micro-CT bone analysis. Bone 2007;41(4):505-15.

[105] Buie HR, Bosma NA, Downey CM, Jirik FR, Boyd SK. Micro-CT evaluation of bone defects: applications to osteolytic bone metastases, bone cysts, and fracture. Med Eng Phys 2013;35(11):1645-50.

[106] Ritman EL. Micro-computed tomography of the lungs and pulmonaryvascular system. Proc Am Thorac Soc 2005;2(6):477-80. 501.

[107] Postnov AA, Meurrens K, Weiler H, Van Dyck D, Xu H, Terpstra P, et al. In vivo assessment of emphysema in mice by high resolution X-ray microtomography. J Microsc 2005;220(Pt 1):70-5.

[108] Shofer S, Badea C, Auerbach S, Schwartz DA, Johnson GA. A micro-computed tomography-based method for the measurement of pulmonary compliance in healthy and bleomycin-exposed mice. Exp Lung Res 2007;33(3-4):169-83.

[109] Guerrero T, Castillo R, Sanders K, Price R, Komaki R, Cody D. Novel method to calculate pulmonary compliance images in rodents from computed tomography acquired at constant pressures. Phys Med Biol 2006;51(5):1101-12.

[110] Guerrero T, Castillo R, Noyola-Martinez J, Torres M, Zhou X, Guerra R, et al. Reduction of pulmonary compliance found with high-resolution computed tomography in irradiated mice. Int J Radiat Oncol Biol Phys 2007;67(3): 879-87.

[111] Saito S, Murase K. Detection and early phase assessment of radiationinduced lung injury in mice using micro-CT. PLoS One 2012;7(9):e45960.

[112] Lam WW, Holdsworth DW, Du LY, Drangova M, McCormack DG, Santyr GE. Micro-CT imaging of rat lung ventilation using continuous image acquisition during xenon gas contrast enhancement. J Appl Physiol 2007;103(5): $1848-56$.

[113] Sangaralingham SJ, Ritman EL, McKie PM, Ichiki T, Lerman A, Scott CG, et al. Cardiac micro-computed tomography imaging of the aging coronary vasculature. Circ Cardiovasc Imaging 2012;5(4):518-24.

[114] Degenhardt K, Wright AC, Horng D, Padmanabhan A, Epstein JA. Rapid 3D phenotyping of cardiovascular development in mouse embryos by micro-CT with iodine staining. Circ Cardiovasc Imaging 2010;3(3):314-22.

[115] Badea CT, Hedlund LW, Mackel JF, Mao L, Rockman HA, Johnson GA. Cardiac micro-computed tomography for morphological and functional phenotyping of muscle LIM protein null mice. Mol Imaging 2007;6(4):261-8.

[116] Nahrendorf M, Badea C, Hedlund LW, Figueiredo JL, Sosnovik DE, Johnson GA, et al. High-resolution imaging of murine myocardial infarction with delayed-enhancement cine micro-CT. Am J Physiol Heart Circ Physiol 2007;292(6):H3172-8.

[117] Badea CT, Wetzel AW, Mistry N, Pomerantz S, Nave D, Johnson GA. Left ventricle volume measurements in cardiac micro-CT: the impact of radiation dose and contrast agent. Comput Med Imaging Graph 2008;32(3):239-50.

[118] Detombe SA, Ford NL, Xiang F, Lu X, Feng Q Drangova M. Longitudina follow-up of cardiac structure and functional changes in an infarct mouse model using retrospectively gated micro-computed tomography. Investig Radiol 2008;43(7):520-9.

[119] Sawall S, Kuntz J, Socher M, Knaup M, Hess A, Bartling S, et al. Imaging of cardiac perfusion of free-breathing small animals using dynamic phasecorrelated micro-CT. Medical Physics 2012;39(12):7499-506.

[120] Haraokawa T, Kido T, Higashino H, Mochizuki T, S. Y. Accuracy of LV volume assessment using cardiac MSCT. In: RSNA; 2004.

[121] Detombe SA, Dunmore-Buyze J, Drangova M. Evaluation of eXIA 160 cardiacrelated enhancement in C57BL/6 and BALB/c mice using micro-CT. Contrast Media Mol Imaging 2012:7(2):240-6.

[122] Yushkevich PA, Piven J, Hazlett HC, Smith RG, Ho S, Gee JC, et al. User-guided 3D active contour segmentation of anatomical structures: significantly improved efficiency and reliability. Neuroimage 2006:31(3):1116-28.

[123] Suri JS. Computer vision, pattern recognition and image processing in left ventricle segmentation: the last 50 years. Pattern Analysis Appl 2000;3(3): 209-42.

[124] Frangi AF, Niessen WJ, Viergever MA. Three-dimensional modeling for functional analysis of cardiac images: a review. IEEE Trans Med Imaging $2001 ; 20(1): 2-25$.

[125] Clark D, Badea A, Johnson GA, Badea CT. Constructing a 4D murine cardiac micro-CT atlas for automated segmentation and phenotyping applications. Proc SPIE 2013;8669. p. 86691P-86691P-12.

[126] Badea CT, Hedlund LW, Cook J, Berridge BR, Johnson GA. Micro-CT imaging assessment of dobutamine-induced cardiac stress in rats. J Pharmacol Toxicol Methods 2011;63(1):24-9.

[127] Badea C, Fubara B, Hedlund L, Johnson G. 4D micro-CT of the mouse heart. Mol Imaging 2005;4(2):110-6.

[128] Badea CT, Johnston SM, Qi Y, Johnson GA. 4D micro-CT for cardiac and perfusion applications with view under sampling. Phys Med Biol 2011; 56(11):3351-69.

[129] Armitage SEJ, Pollmann SI, Detombe SA, Drangova M. Least-error projection sorting to optimize retrospectively gated cardiac micro-CT of free-breathing mice. Med Phys 2012;39(3):1452-61.
[130] Johnston SM, Johnson GA, Badea CT. Temporal and spectral imaging with micro-CT. Med Phys 2012;39(8):4943-58.

[131] Cody DD, Nelson CL, Bradley WM, Wislez M, Juroske D, Price RE, et al. Murine lung tumor measurement using respiratory-gated micro-computed tomography. Invest Radiol 2005;40(5):263-9.

[132] Kan Z, Phongkitkarun S, Kobayashi S, Tang Y, Ellis LM, Lee TY, et al. Functional CT for quantifying tumor perfusion in antiangiogenic therapy in a rat model. Radiology 2005;237(1):151-8.

[133] Phongkitkarun S, Kobayashi S, Kan Z, Lee TY, Charnsangavej C. Quantification of angiogenesis by functional computed tomography in a Matrigel model in rats. Acad Radiol 2004;11(5):573-82.

[134] Du LY, Umoh J, Nikolov HN, Pollmann SI, Lee TY, Holdsworth DW. A quality assurance phantom for the performance evaluation of volumetric micro-CT systems. Phys Med Biol 2007;52(23):7087-108.

[135] Kindlmann GL, Weinstein DM, Jones GM, Johnson CR, Capecchi MR, Keller C. Practical vessel imaging by computed tomography in live transgenic mouse models for human tumors. Mol Imaging 2005;4(4):417-24.

[136] Almajdub M, Nejjari M, Poncet G, Magnier L, Chereul E, Roche C, et al. In-vivo high-resolution X-ray microtomography for liver and spleen tumor assessment in mice. Contrast Media Mol Imaging 2007;2(2):88-93.

[137] Suckow CE, Stout DB. MicroCT liver contrast agent enhancement over time, dose, and mouse strain. Mol Imaging Biol 2008;10(2):114-20.

[138] Graham KC, Ford NL, MacKenzie LT, Postenka CO, Groom AC, MacDonald IC, et al. Noninvasive quantification of tumor volume in preclinical liver metastasis models using contrast-enhanced $\mathrm{x}$-ray computed tomography. Invest Radiol 2008;43(2):92-9.

[139] Boll H, Nittka S, Doyon F, Neumaier M, Marx A, Kramer M, et al. Micro-CT based experimental liver imaging using a nanoparticulate contrast agent: a longitudinal study in mice. Plos One 2011;6(9).

[140] Ghaghada KB, Badea CT, Karumbaiah L, Fettig N, Bellamkonda RV, Johnson GA, et al. Evaluation of tumor microenvironment in an animal model using a nanoparticle contrast agent in computed tomography imaging. Acad Radiol 2011;18(1):20-30.

[141] Moding EJ, Clark DP, Qi Y, Li Y, Ma Y, Ghaghada K, et al. Dual-energy microcomputed tomography imaging of radiation-induced vascular changes in primary mouse sarcomas. Int J Radiat Oncol Biol Phys 2013;85(5):1353-9.

[142] Maeda $H$. The enhanced permeability and retention (EPR) effect in tumor vasculature: the key role of tumor-selective macromolecular drug targeting. Adv Enzyme Regul 2001;41:189-207.

[143] Badea CT, Athreya KK, Espinosa G, Clark D, Ghafoori AP, Li Y, et al. Computed tomography imaging of primary lung cancer in mice using a liposomaliodinated contrast agent. PLoS ONE 2012;7(4):e34496.

[144] Kepshire D, Mincu N, Hutchins M, Gruber J, Dehghani H, Hypnarowski J, et al. A microcomputed tomography guided fluorescence tomography system for small animal molecular imaging. Rev Sci Instrum 2009;80(4):043701.

[145] Goertzen AL, Meadors AK, Silverman RW, Cherry SR. Simultaneous molecular and anatomical imaging of the mouse in vivo. Phys Med Biol 2002;47(24): 4315-28.

[146] Kastis GA, Furenlid LR, Wilson DW, Peterson TE, Barber HB, Barrett HH. Compact CT/SPECT Small-Animal Imaging System. IEEE Trans Nucl Sci 2004:51:63-7.

[147] Liang H, Yang Y, Yang K, Wu Y, Boone JM, Cherry SR. A microPET/CT system for in vivo small animal imaging. Phys Med Biol 2007;52(13):3881-94.

[148] Jan ML, Chuang KS, Chen GW, Ni YC, Chen S, Chang CH, et al. A threedimensional registration method for automated fusion of micro PET-CTSPECT whole-body images. IEEE Trans Med Imaging 2005;24(7):886-93.

[149] Chow PL, Rannou FR, Chatziioannou AF. Attenuation correction for small animal PET tomographs. Phys Med Biol 2005;50(8):1837-50.

[150] Chang L-T. A Method for attenuation correction in radionuclide computed tomography. Nucl Sci IEEE Trans 1978;25(1):638-43.

[151] Hwang AB, Hasegawa BH. Attenuation correction for small animal SPECT imaging using x-ray CT data. Med Phys 2005;32(9):2799-804.

[152] Vanhove C, Defrise M, Bossuyt A, Lahoutte T. Improved quantification in single-pinhole and multiple-pinhole SPECT using micro-CT information. Eur J Nucl Med Mol Imaging 2009:36(7):1049-63.

[153] Beekman FJ, van der Have F, Vastenhouw B, van der Linden AJ, van Rijk PP, Burbach JP, et al. U-SPECT-I: a novel system for submillimeter-resolution tomography with radiolabeled molecules in mice. J Nucl Med 2005;46(7): 1194-200.

[154] Tai JH, Nguyen B, Wells RG, Kovacs MS, McGirr R, Prato FS, et al. Imaging of gene expression in live pancreatic islet cell lines using dual-isotope SPECT. J Nucl Med 2008:49(1):94-102.

[155] Ritman EL. Molecular imaging in small animals-roles for micro-CT. J Cell Biochem Suppl 2002;39:116-24.

[156] Badea C, Befera N, Clark D, Qi Y, Johnson G. Dual-energy micro-CT imaging of pulmonary airway obstruction: correlation with micro-SPECT. SPIE Med Imaging 2014;9038. p. 90380U-90380U-8.

[157] Fornaro J, Leschka S, Hibbeln D, Butler A, Anderson N, Pache G, et al. Dualand multi-energy CT: approach to functional imaging. Insights Imaging 2011:2(2):149-59.

[158] Taschereau R, Chatziioannou AF. Preclinical dual-energy x-ray computed tomography through differential filtration. In: Nuclear Science Symposium Conference Record, 2008. NSS '08. IEEE; 2008.

[159] Guo X, Johnston SM, Johnson GA, Badea CT. A comparison of sampling strategies for dual energy micro-CT. Proc SPIE 2012;8313:831332. 
[160] Ashton JR, Clark DP, Moding EJ, Ghaghada K, Kirsch DG, West JL, et al. Dualenergy micro-ct functional imaging of primary lung cancer in mice using gold and iodine nanoparticle contrast agents: a validation study. PLoS One 2014;9(2):e88129.

[161] Badea C, Johnston S, Qi Y, Ghaghada K, Johnson G. Dual-energy micro-CT imaging for differentiation of iodine-and gold-based nanoparticles. Proc SPIE 2011;7961:79611X

[162] Badea CT, Guo X, Clark D, Johnston SM, Marshall CD, Piantadosi CA. Dualenergy micro-CT of the rodent lung. Am J Physiol Lung Cell Mol Physiol 2012;302(10):L1088-97.

[163] Lee CL, Min H, Befera N, Clark D, Qi Y, Das S, et al. Assessing cardiac injury in mice with dual energy-Microct, 4D-Microct, and MicroSPECT imaging after partial heart irradiation. Int J Radiat Oncol Biol Phys 2014; 88(3):686-93.

[164] Moding EJ, Clark DP, Qi Y, Li Y, Ma Y, Ghaghada K, et al. Dual-Energy microcomputed tomography imaging of radiation-induced vascular changes in primary mouse sarcomas. Int J Radiat Oncol Biol Phys 2012.

[165] Clark DP, Ghaghada K, Moding EJ, Kirsch DG, Badea CT. In vivo characterization of tumor vasculature using iodine and gold nanoparticles and dual energy micro-CT. Phys Med Biol 2013·58(6):1683-704.

[166] Bhavane R, Badea C, Ghaghada KB, Clark D, Vela D, Moturu A, et al. Dualenergy computed tomography imaging of atherosclerotic plaques in a mouse model using a liposomal-iodine nanoparticle contrast agent. Circ Cardiovasc Imaging 2013;6(2):285-94.

[167] Granton PV, Pollmann SI, Ford NL, Drangova M, Holdsworth DW. Implementation of dual- and triple-energy cone-beam micro-CT for postreconstruction material decomposition. Med Phys 2008;35(11):5030-42.

[168] Schlomka JP, Roessl E, Dorscheid R, Dill S, Martens G, Istel T, et al. Experimental feasibility of multi-energy photon-counting K-edge imaging in preclinical computed tomography. Phys Med Biol 2008;53(15):4031-47.

[169] Xu Q, Yu H, Bennett J, He P, Zainon R, Doesburg R, et al. Image reconstruction for hybrid true-color micro-CT. IEEE Trans Biomed Eng 2012;59(6):1711-9.

[170] Hainfeld JF, Dilmanian FA, Slatkin DN, Smilowitz HM. Radiotherapy enhancement with gold nanoparticles. J Pharm Pharmacol 2008;60(8): 977-85.

[171] Hainfeld JF, Dilmanian FA, Zhong Z, Slatkin DN, Kalef-Ezra JA, Smilowitz HM. Gold nanoparticles enhance the radiation therapy of a murine squamous cell carcinoma. Phys Med Biol 2010;55(11):3045-59.

[172] Hainfeld JF, Slatkin DN, Smilowitz HM. The use of gold nanoparticles to enhance radiotherapy in mice. Phys Med Biol 2004;49(18):N309-15.

[173] Bravin A, Coan P, Suortti P. X-ray phase-contrast imaging: from pre-clinical applications towards clinics. Phys Med Biol 2013;58(1):R1.

[174] Langer M, Cloetens P, Guigay J-P, Peyrin F. Quantitative comparison of direct phase retrieval algorithms in in-line phase tomography. Med Phys 2008;35(10):4556-66.

[175] Chapman D, Thomlinson W, Johnston R, Washburn D, Pisano E, Gmür N, et al. Diffraction enhanced x-ray imaging. Phys Med Biol 1997;42(11):2015.

[176] David C, Weitkamp T, Pfeiffer F, Diaz A, Bruder J, Rohbeck T, et al. Hard X-ray phase imaging and tomography using a grating interferometer. Spectrochim Acta Part B At Spectrosc 2007;62(6):626-30.
[177] Pfeiffer F, Weitkamp T, Bunk O, David C. Phase retrieval and differential phase-contrast imaging with low-brilliance X-ray sources. Nat Phys 2006;2(4):258-61.

[178] Bech M, Tapfer A, Velroyen A, Yaroshenko A, Pauwels B, Hostens J, et al. Invivo dark-field and phase-contrast X-ray imaging. Sci Reports 2013:3.

[179] Pauwels B, Bruyndonckxa P, Liua X, Tapferb A, Velroyenb A, Yaroshenkob A et al. First small-animal in-vivo phase-contrast micro-CT scanner. In: SPIE optical engineering + applications, 8506; 2012. p. 85060J-85060J-8.

[180] Tapfer A, Bech M, Velroyen A, Meiser J, Mohr J, Walter M, et al. Experimenta results from a preclinical X-ray phase-contrast CT scanner. Proc Natl Acad Sci 2012;109(39):15691-6.

[181] Tapfer A, Braren R, Bech M, Willner M, Zanette I, Weitkamp T, et al. X-ray phase-contrast CT of a pancreatic ductal adenocarcinoma mouse model. PloS one 2013;8(3):e58439.

[182] Willner M, Herzen J, Grandl S, Auweter S, Mayr D, Hipp A, et al. Quantitative breast tissue characterization using grating-based x-ray phase-contrast imaging. Phys Med Biology 2014;59(7):1557.

[183] Meinel FG, Schwab F, Yaroshenko A, Velroyen A, Bech M, Hellbach K, et al. Lung tumors on multimodal radiographs derived from grating-based X-ray imaging - a feasibility study. Phys Med 2014;30(3):352-7.

[184] Munro PR, Ignatyev K, Speller RD, Olivo A. Phase and absorption retrieva using incoherent X-ray sources. Proc Natl Acad Sci 2012;109(35):13922-7.

185] Connor D, Pisano E, Cole E, Zhong Z, Dilmanian F, Parham C, et al. Translation of synchrotron-based research into the clinic: assessing the current clinical potential of diffraction enhanced imaging. Synchrotron Radiat News $2011 ; 24(2): 29-33$.

[186] Fu J Willner M, Chen L Tan R, Achterhold K, Bech M, et al. Helical differentia X-ray phase-contrast computed tomography. Phys Med 2014;30(3):374-9.

[187] Bevins N, Zambelli J, Li K, Qi Z, Chen G-H. Multicontrast X-ray computed tomography imaging using Talbot-Lau interferometry without phase stepping. Med Phys 2011;39(1):424-8.

[188] Epple F, Potdevin G, Thibault P, Ehn S, Herzen J, Hipp A, et al. Unwrapping differential $\mathrm{X}$-ray phase-contrast images through phase estimation from multiple energy data. Opt Express 2013:21(24):29101-8.

[189] Stutman D, Stayman J, Finkenthal M, Siewerdsen J. High Energy X-ray PhaseContrast Imaging Using Glancing Angle Grating Interferometers. Proc SPIE 2013;8668. p. 866814-1.

[190] Köhler T, Brendel B, Roessl E. Iterative reconstruction for differential phase contrast imaging using spherically symmetric basis functions. Med Phys $2011 ; 38(8): 4542-5$

[191] Fu J, Schleede S, Tan R, Chen L, Bech M, Achterhold K, et al. An algebraic iterative reconstruction technique for differential X-ray phase-contrast computed tomography. Zeitschrift für Medizinische Physik 2013;23(3) 186-93.

[192] Herzen J, Willner MS, Fingerle AA, Noël PB, Köhler T, Drecoll E, et al. Imaging liver lesions using grating-based phase-contrast computed tomography with bi-lateral filter post-processing. PloS one 2014;9(1):e83369.

[193] Nilchian M, Unser M. Differential phase-contrast X-ray computed tomography: from model discretization to image reconstruction. In: Biomedica Imaging (ISBI), 2012 9th IEEE International Symposium on. IEEE; 2012. 